\title{
Unified Gauge Theories and Reduction of Couplings: from Finiteness to Fuzzy Extra Dimensions ${ }^{\star}$
}

\author{
Myriam MONDRAGÓN ${ }^{\dagger}$ and George ZOUPANOS $\ddagger$ \\ † Inst. de Física, Universidad Nacional Autónoma de México, México \\ E-mail:myriam@fisica.unam.mx \\ $¥$ Physics Department, National Technical University, Athens, Greece \\ E-mail: george.zoupanos@cern.ch
}

Received November 01, 2007, in final form January 31, 2008; Published online February 23, 2008

Original article is available at http://www.emis.de/journals/SIGMA/2008/026/

\begin{abstract}
Finite Unified Theories (FUTs) are $N=1$ supersymmetric Grand Unified Theories, which can be made all-loop finite, both in the dimensionless (gauge and Yukawa couplings) and dimensionful (soft supersymmetry breaking terms) sectors. This remarkable property, based on the reduction of couplings at the quantum level, provides a drastic reduction in the number of free parameters, which in turn leads to an accurate prediction of the top quark mass in the dimensionless sector, and predictions for the Higgs boson mass and the supersymmetric spectrum in the dimensionful sector. Here we examine the predictions of two such FUTs. Next we consider gauge theories defined in higher dimensions, where the extra dimensions form a fuzzy space (a finite matrix manifold). We reinterpret these gauge theories as four-dimensional theories with Kaluza-Klein modes. We then perform a generalized à la Forgacs-Manton dimensional reduction. We emphasize some striking features emerging such as (i) the appearance of non-Abelian gauge theories in four dimensions starting from an Abelian gauge theory in higher dimensions, (ii) the fact that the spontaneous symmetry breaking of the theory takes place entirely in the extra dimensions and (iii) the renormalizability of the theory both in higher as well as in four dimensions. Then reversing the above approach we present a renormalizable four dimensional $S U(N)$ gauge theory with a suitable multiplet of scalar fields, which via spontaneous symmetry breaking dynamically develops extra dimensions in the form of a fuzzy sphere $S_{N}^{2}$. We explicitly find the tower of massive Kaluza-Klein modes consistent with an interpretation as gauge theory on $M^{4} \times S^{2}$, the scalars being interpreted as gauge fields on $S^{2}$. Depending on the parameters of the model the low-energy gauge group can be $S U(n)$, or broken further to $S U\left(n_{1}\right) \times S U\left(n_{2}\right) \times U(1)$. Therefore the second picture justifies the first one in a renormalizable framework but in addition has the potential to reveal new aspects of the theory.
\end{abstract}

Key words: unification; gauge theories; finiteness; higher dimensions; fuzzy sphere; noncommutative gauge theories; renormalizability

2000 Mathematics Subject Classification: 81T60; 81V22; 83E15; 81T75; 54A40

\section{Introduction}

The theoretical efforts to establish a deeper understanding of Nature have led to very interesting frameworks such as String theories and Non-commutative Geometry both of which aim to describe physics at the Planck scale. Looking for the origin of the idea that coordinates might not commute we might have to go back to the days of Heisenberg. In the recent years the birth of such speculations can be found in $[1,2]$. In the spirit of Non-commutative Geometry

${ }^{\star}$ This paper is a contribution to the Proceedings of the Seventh International Conference "Symmetry in Nonlinear Mathematical Physics" (June 24-30, 2007, Kyiv, Ukraine). The full collection is available at http://www.emis.de/journals/SIGMA/symmetry2007.html 
also particle models with non-commutative gauge theory were explored [3] (see also [4]), [5, 6]. On the other hand the present intensive research has been triggered by the natural realization of non-commutativity of space in the string theory context of D-branes in the presence of a constant background antisymmetric field [7]. After the work of Seiberg and Witten [8], where a map (SW map) between non-commutative and commutative gauge theories has been described, there has been a lot of activity also in the construction of non-commutative phenomenological Lagrangians, for example various non-commutative standard model like Lagrangians have been proposed $[9,10]^{1}$. In particular in [10], following the SW map methods developed in [11], a noncommutative standard model with $S U(3) \times S U(2) \times U(1)$ gauge group has been presented. These non-commutative models represent interesting generalizations of the SM and hint at possible new physics. However they do not address the usual problem of the SM, the presence of a plethora of free parameters mostly related to the ad hoc introduction of the Higgs and Yukawa sectors in the theory. At this stage it is worth recalling that various schemes, with the Coset Space Dimensional Reduction (CSDR) [14, 15, 16, 17] being pioneer, were suggesting that a unification of the gauge and Higgs sectors can be achieved in higher dimensions. Moreover the addition of fermions in the higher-dimensional gauge theory leads naturally after CSDR to Yukawa couplings in four dimensions. In the successes of the CSDR scheme certainly should be added the possibility to obtain chiral theories in four dimensions [18, 19, 20, 21] as well as softly broken supersymmetric or non-supersymmetric theories starting from a supersymmetric gauge theory defined in higher dimensions [22].

The original plan of this paper was to present an overview covering the following subjects:

a) Quantum Reduction of Couplings and Finite Unified Theories;

b) Classical Reduction of Couplings and Coset Space Dimensional Reduction;

c) Renormalizable Unified Theories from Fuzzy Higher Dimensions [23].

The aim was to present an unified description of our current attempts to reduce the free parameters of the Standard Model by using Finite Unification and extra dimensions. However we will cover only the first and the third subjects given the fact that there exists extensive reviews covering a major part of the second one [15, 16]. These two topics represent different attempts at reduction of couplings, on one hand the Finite Unified Theories showing promising models with good phenomenology, on the other hand, the Unified Theories from Fuzzy Higher Dimensions combining dimensional reduction and reduction of couplings in a renormalizable theory.

\section{Reduction of Couplings and Finiteness in $N=1$ SUSY Gauge Theories}

Finite Unified Theories are $N=1$ supersymmetric Grand Unified Theories (GUTs) which can be made finite even to all-loop orders, including the soft supersymmetry breaking sector. The method to construct GUTs with reduced independent parameters [24, 25] consists of searching for renormalization group invariant (RGI) relations holding below the Planck scale, which in turn are preserved down to the GUT scale. Of particular interest is the possibility to find RGI relations among couplings that guarantee finitenes to all-orders in perturbation theory [26, 27]. In order to achieve the latter it is enough to study the uniqueness of the solutions to the oneloop finiteness conditions [26, 27]. The constructed finite unified $N=1$ supersymmetric $S U(5)$

\footnotetext{
${ }^{1}$ These SM actions are mainly considered as effective actions because they are not renormalizable. The effective action interpretation is consistent with the SM in [10] being anomaly free [12]. Non-commutative phenomenology has been discussed in [13].
} 
GUTs, using the above tools, predicted correctly from the dimensionless sector (gauge-Yukawa unification), among others, the top quark mass [28]. The search for RGI relations and finiteness has been extended to the soft supersymmetry breaking sector (SSB) of these theories [29, 30], which involves parameters of dimension one and two. Eventually, the full theories can be made all-loop finite and their predictive power is extended to the Higgs sector and the supersymmetric spectrum (s-spectrum).

Here let us review the main points and ideas concerning the reduction of couplings and finiteness in $N=1$ supersymmetric theories. A RGI relation among couplings $g_{i}, \Phi\left(g_{1}, \ldots, g_{N}\right)=0$, has to satisfy the partial differential equation $\mu d \Phi / d \mu=\sum_{i=1}^{N} \beta_{i} \partial \Phi / \partial g_{i}=0$, where $\beta_{i}$ is the $\beta$-function of $g_{i}$. There exist $(N-1)$ independent $\Phi$ 's, and finding the complete set of these solutions is equivalent to solve the so-called reduction equations (REs) [25], $\beta_{g}\left(d g_{i} / d g\right)=\beta_{i}$, $i=1, \ldots, N$, where $g$ and $\beta_{g}$ are the primary coupling and its $\beta$-function. Using all the $(N-1) \Phi$ 's to impose RGI relations, one can in principle express all the couplings in terms of a single coupling $g$. The complete reduction, which formally preserves perturbative renormalizability, can be achieved by demanding a power series solution, whose uniqueness can be investigated at the one-loop level.

Finiteness can be understood by considering a chiral, anomaly free, $N=1$ globally supersymmetric gauge theory based on a group $\mathrm{G}$ with gauge coupling constant $g$. The superpotential of the theory is given by

$$
W=\frac{1}{2} m^{i j} \Phi_{i} \Phi_{j}+\frac{1}{6} C^{i j k} \Phi_{i} \Phi_{j} \Phi_{k},
$$

where $m^{i j}$ (the mass terms) and $C^{i j k}$ (the Yukawa couplings) are gauge invariant tensors and the matter field $\Phi_{i}$ transforms according to the irreducible representation $R_{i}$ of the gauge group $G$.

The one-loop $\beta$-function of the gauge coupling $g$ is given by

$$
\beta_{g}^{(1)}=\frac{d g}{d t}=\frac{g^{3}}{16 \pi^{2}}\left[\sum_{i} T\left(R_{i}\right)-3 C_{2}(G)\right],
$$

where $T\left(R_{i}\right)$ is the Dynkin index of $R_{i}$ and $C_{2}(G)$ is the quadratic Casimir of the adjoint representation of the gauge group $G$. The $\beta$-functions of $C^{i j k}$, by virtue of the non-renormalization theorem, are related to the anomalous dimension matrix $\gamma_{i}^{j}$ of the matter fields $\Phi_{i}$ as:

$$
\beta_{C}^{i j k}=\frac{d}{d t} C^{i j k}=C^{i j p} \sum_{n=1} \frac{1}{\left(16 \pi^{2}\right)^{n}} \gamma_{p}^{k(n)}+(k \leftrightarrow i)+(k \leftrightarrow j) .
$$

At one-loop level $\gamma_{i}^{j}$ is given by

$$
\gamma_{i}^{j(1)}=\frac{1}{2} C_{i p q} C^{j p q}-2 g^{2} C_{2}\left(R_{i}\right) \delta_{i}^{j},
$$

where $C_{2}\left(R_{i}\right)$ is the quadratic Casimir of the representation $R_{i}$, and $C^{i j k}=C_{i j k}^{*}$.

All the one-loop $\beta$-functions of the theory vanish if the $\beta$-function of the gauge coupling $\beta_{g}^{(1)}$, and the anomalous dimensions $\gamma_{i}^{j(1)}$, vanish, i.e.

$$
\sum_{i} T\left(R_{i}\right)=3 C_{2}(G), \quad \frac{1}{2} C_{i p q} C^{j p q}=2 \delta_{i}^{j} g^{2} C_{2}\left(R_{i}\right) .
$$

The conditions for finiteness for $N=1$ field theories with $S U(N)$ gauge symmetry are discussed in [31], and the analysis of the anomaly-free and no-charge renormalization requirements for these theories can be found in [32]. A very interesting result is that the conditions (2.2) are necessary and sufficient for finiteness at the two-loop level [33]. 
The one- and two-loop finiteness conditions (2.2) restrict considerably the possible choices of the irreducible representations $R_{i}$ for a given group $G$ as well as the Yukawa couplings in the superpotential (2.1). Note in particular that the finiteness conditions cannot be applied to the supersymmetric standard model (SSM), since the presence of a $U(1)$ gauge group is incompatible with the condition $(2.2)$, due to $C_{2}[U(1)]=0$. This leads to the expectation that finiteness should be attained at the grand unified level only, the SSM being just the corresponding low-energy, effective theory.

The finiteness conditions impose relations between gauge and Yukawa couplings. Therefore, we have to guarantee that such relations leading to a reduction of the couplings hold at any renormalization point. The necessary, but also sufficient, condition for this to happen is to require that such relations are solutions to the reduction equations (REs) to all orders. The all-loop order finiteness theorem of [26] is based on: (a) the structure of the supercurrent in $N=1 \mathrm{SYM}$ and on (b) the non-renormalization properties of $N=1$ chiral anomalies [26]. Alternatively, similar results can be obtained [27, 36] using an analysis of the all-loop NSVZ gauge beta-function [37].

\section{Soft supersymmetry breaking and finiteness}

The above described method of reducing the dimensionless couplings has been extended [30, 29] to the soft supersymmetry breaking (SSB) dimensionful parameters of $N=1$ supersymmetric theories. Recently very interesting progress has been made [38, 39, 40, 41, 42, 43, 44, 45, 46] concerning the renormalization properties of the SSB parameters, based conceptually and technically on the work of [40]. In this work the powerful supergraph method [43] for studying supersymmetric theories has been applied to the softly broken ones by using the "spurion" external space-time independent superfields [44]. In the latter method a softly broken supersymmetric gauge theory is considered as a supersymmetric one in which the various parameters such as couplings and masses have been promoted to external superfields that acquire "vacuum expectation values". Based on this method the relations among the soft term renormalization and that of an unbroken supersymmetric theory have been derived. In particular the $\beta$-functions of the parameters of the softly broken theory are expressed in terms of partial differential operators involving the dimensionless parameters of the unbroken theory. The key point in the strategy of $[38,39,40,41,42,43,44,45,46]$ in solving the set of coupled differential equations so as to be able to express all parameters in a RGI way, was to transform the partial differential operators involved to total derivative operators [38]. It is indeed possible to do this on the RGI surface which is defined by the solution of the reduction equations. In addition it was found that RGI SSB scalar masses in gauge-Yukawa unified models satisfy a universal sum rule at one-loop [42]. This result was generalized to two-loops for finite theories [46], and then to all-loops for general gauge-Yukawa and Finite Unified Theories [39].

In order to obtain a feeling of some of the above results, consider the superpotential given by (2.1) along with the Lagrangian for SSB terms

$$
-\mathcal{L}_{\mathrm{SB}}=\frac{1}{6} h^{i j k} \phi_{i} \phi_{j} \phi_{k}+\frac{1}{2} b^{i j} \phi_{i} \phi_{j}+\frac{1}{2}\left(m^{2}\right)_{i}^{j} \phi^{* i} \phi_{j}+\frac{1}{2} M \lambda \lambda+\text { H.c. },
$$

where the $\phi_{i}$ are the scalar parts of the chiral superfields $\Phi_{i}, \lambda$ are the gauginos and $M$ their unified mass. Since only finite theories are considered here, it is assumed that the gauge group is a simple group and the one-loop $\beta$-function of the gauge coupling $g$ vanishes. It is also assumed that the reduction equations admit power series solutions of the form

$$
C^{i j k}=g \sum_{n=0} \rho_{(n)}^{i j k} g^{2 n}
$$


According to the finiteness theorem [26], the theory is then finite to all-orders in perturbation theory, if, among others, the one-loop anomalous dimensions $\gamma_{i}^{j(1)}$ vanish. The one- and two-loop finiteness for $h^{i j k}$ can be achieved by [35]

$$
h^{i j k}=-M C^{i j k}+\cdots=-M \rho_{(0)}^{i j k} g+O\left(g^{5}\right) .
$$

An additional constraint in the SSB sector up to two-loops [46], concerns the soft scalar masses as follows

$$
\frac{\left(m_{i}^{2}+m_{j}^{2}+m_{k}^{2}\right)}{M M^{\dagger}}=1+\frac{g^{2}}{16 \pi^{2}} \Delta^{(2)}+O\left(g^{4}\right)
$$

for $i, j, k$ with $\rho_{(0)}^{i j k} \neq 0$, where $\Delta^{(2)}$ is the two-loop correction

$$
\Delta^{(2)}=-2 \sum_{l}\left[\left(m_{l}^{2} / M M^{\dagger}\right)-(1 / 3)\right] T\left(R_{l}\right),
$$

which vanishes for the universal choice [35], i.e. when all the soft scalar masses are the same at the unification point.

If we know higher-loop $\beta$-functions explicitly, we can follow the same procedure and find higher-loop RGI relations among SSB terms. However, the $\beta$-functions of the soft scalar masses are explicitly known only up to two loops. In order to obtain higher-loop results, we need something else instead of knowledge of explicit $\beta$-functions, e.g. some relations among $\beta$-functions.

The recent progress made using the spurion technique $[43,44]$ leads to the following all-loop relations among SSB $\beta$-functions [38, 39, 40, 41, 42, 43, 44, 45, 46]

$$
\begin{aligned}
& \beta_{M}=2 \mathcal{O}\left(\frac{\beta_{g}}{g}\right) \\
& \beta_{h}^{i j k}=\gamma^{i}{ }_{l} h^{l j k}+\gamma^{j}{ }_{l} h^{i l k}+\gamma^{k}{ }_{l} h^{i j l}-2 \gamma_{1 l}^{i} C^{l j k}-2 \gamma_{1 l}^{j} C^{i l k}-2 \gamma_{1 l}^{k} C^{i j l}, \\
& \left(\beta_{m^{2}}{ }^{i}{ }_{j}=\left[\Delta+X \frac{\partial}{\partial g}\right] \gamma^{i}{ }_{j},\right. \\
& \mathcal{O}=\left(M g^{2} \frac{\partial}{\partial g^{2}}-h^{l m n} \frac{\partial}{\partial C^{l m n}}\right), \\
& \Delta=2 \mathcal{O O}^{*}+2|M|^{2} g^{2} \frac{\partial}{\partial g^{2}}+\tilde{C}_{l m n} \frac{\partial}{\partial C_{l m n}}+\tilde{C}^{l m n} \frac{\partial}{\partial C^{l m n}}
\end{aligned}
$$

where $\left(\gamma_{1}\right)^{i}{ }_{j}=\mathcal{O} \gamma^{i}{ }_{j}, C_{l m n}=\left(C^{l m n}\right)^{*}$, and

$$
\tilde{C}^{i j k}=\left(m^{2}\right)^{i}{ }_{l} C^{l j k}+\left(m^{2}\right)^{j}{ }_{l} C^{i l k}+\left(m^{2}\right)^{k}{ }_{l} C^{i j l} .
$$

It was also found [45] that the relation

$$
h^{i j k}=-M\left(C^{i j k}\right)^{\prime} \equiv-M \frac{d C^{i j k}(g)}{d \ln g},
$$

among couplings is all-loop RGI. Furthermore, using the all-loop gauge $\beta$-function of Novikov et al. [37] given by

$$
\beta_{g}^{\mathrm{NSVZ}}=\frac{g^{3}}{16 \pi^{2}}\left[\frac{\sum_{l} T\left(R_{l}\right)\left(1-\gamma_{l} / 2\right)-3 C(G)}{1-g^{2} C(G) / 8 \pi^{2}}\right],
$$


it was found the all-loop RGI sum rule [39],

$$
\begin{aligned}
m_{i}^{2}+m_{j}^{2}+m_{k}^{2}= & |M|^{2}\left\{\frac{1}{1-g^{2} C(G) /\left(8 \pi^{2}\right)} \frac{d \ln C^{i j k}}{d \ln g}+\frac{1}{2} \frac{d^{2} \ln C^{i j k}}{d(\ln g)^{2}}\right\} \\
& +\sum_{l} \frac{m_{l}^{2} T\left(R_{l}\right)}{C(G)-8 \pi^{2} / g^{2}} \frac{d \ln C^{i j k}}{d \ln g} .
\end{aligned}
$$

In addition the exact- $\beta$-function for $m^{2}$ in the NSVZ scheme has been obtained [39] for the first time and is given by

$$
\begin{aligned}
\beta_{m_{i}^{2}}^{\mathrm{NSVZ}}= & {\left[|M|^{2}\left\{\frac{1}{1-g^{2} C(G) /\left(8 \pi^{2}\right)} \frac{d}{d \ln g}+\frac{1}{2} \frac{d^{2}}{d(\ln g)^{2}}\right\}\right.} \\
& \left.+\sum_{l} \frac{m_{l}^{2} T\left(R_{l}\right)}{C(G)-8 \pi^{2} / g^{2}} \frac{d}{d \ln g}\right] \gamma_{i}^{\mathrm{NSVZ}} .
\end{aligned}
$$

\section{Finite Unified Theories}

The first one- and two-loop $S U(5)$ finite model was presented in [47]. Here we will examine Finite Unified Theories with $S U(5)$ gauge group, where the reduction of couplings has been applied to the third generation of quarks and leptons. An extension to three families, and the generation of quark mixing angles and masses in Finite Unified Theories has been addressed in [48], where several examples are given. These extensions are not considered here. Realistic Finite Unified Theories based on product gauge groups, where the finiteness implies three generations of matter, have also been studied [49].

A predictive gauge-Yukawa unified $S U(5)$ model which is finite to all orders, in addition to the requirements mentioned already, should also have the following properties:

1. One-loop anomalous dimensions are diagonal, i.e., $\gamma_{i}^{(1) j} \propto \delta_{i}^{j}$.

2. Three fermion generations, in the irreducible representations $\overline{\mathbf{5}}_{i}, \mathbf{1 0}_{i}(i=1,2,3)$, which obviously should not couple to the adjoint $\mathbf{2 4}$.

3. The two Higgs doublets of the MSSM should mostly be made out of a pair of Higgs quintet and anti-quintet, which couple to the third generation.

In the following we discuss two versions of the all-order finite model. The model of [28], which will be labeled $\mathbf{A}$, and a slight variation of this model (labeled $\mathbf{B}$ ), which can also be obtained from the class of the models suggested in [38] with a modification to suppress non-diagonal anomalous dimensions.

The superpotential which describes the two models takes the form $[28,46]$

$$
\begin{aligned}
W= & \sum_{i=1}^{3}\left[\frac{1}{2} g_{i}^{u} \mathbf{1 0}_{i} \mathbf{1 0}_{i} H_{i}+g_{i}^{d} \mathbf{1 0}_{i} \overline{\mathbf{5}}_{i} \bar{H}_{i}\right]+g_{23}^{u} \mathbf{1 0}_{2} \mathbf{1 0}_{3} H_{4}+g_{23}^{d} \mathbf{1 0}_{2} \overline{\mathbf{5}}_{3} \bar{H}_{4} \\
& +g_{32}^{d} \mathbf{1 0}_{3} \overline{\mathbf{5}}_{2} \bar{H}_{4}+\sum_{a=1}^{4} g_{a}^{f} H_{a} \mathbf{2 4} \bar{H}_{a}+\frac{g^{\lambda}}{3}(\mathbf{2 4})^{3},
\end{aligned}
$$

where $H_{a}$ and $\bar{H}_{a}(a=1, \ldots, 4)$ stand for the Higgs quintets and anti-quintets.

The non-degenerate and isolated solutions to $\gamma_{i}^{(1)}=0$ for the models $\{\mathbf{A}, \mathbf{B}\}$ are:

$$
\begin{aligned}
& \left(g_{1}^{u}\right)^{2}=\left\{\frac{8}{5}, \frac{8}{5}\right\} g^{2}, \quad\left(g_{1}^{d}\right)^{2}=\left\{\frac{6}{5}, \frac{6}{5}\right\} g^{2}, \quad\left(g_{2}^{u}\right)^{2}=\left(g_{3}^{u}\right)^{2}=\left\{\frac{8}{5}, \frac{4}{5}\right\} g^{2}, \\
& \left(g_{2}^{d}\right)^{2}=\left(g_{3}^{d}\right)^{2}=\left\{\frac{6}{5}, \frac{3}{5}\right\} g^{2}, \quad\left(g_{23}^{u}\right)^{2}=\left\{0, \frac{4}{5}\right\} g^{2}, \quad\left(g_{23}^{d}\right)^{2}=\left(g_{32}^{d}\right)^{2}=\left\{0, \frac{3}{5}\right\} g^{2}, \\
& \left(g^{\lambda}\right)^{2}=\frac{15}{7} g^{2}, \quad\left(g_{2}^{f}\right)^{2}=\left(g_{3}^{f}\right)^{2}=\left\{0, \frac{1}{2}\right\} g^{2}, \quad\left(g_{1}^{f}\right)^{2}=0, \quad\left(g_{4}^{f}\right)^{2}=\{1,0\} g^{2} .
\end{aligned}
$$


According to the theorem of [26] these models are finite to all orders. After the reduction of couplings the symmetry of $W$ is enhanced [28, 46].

The main difference of the models $\mathbf{A}$ and $\mathbf{B}$ is that two pairs of Higgs quintets and antiquintets couple to the $\mathbf{2 4}$ for $\mathbf{B}$ so that it is not necessary to mix them with $H_{4}$ and $\bar{H}_{4}$ in order to achieve the triplet-doublet splitting after the symmetry breaking of $S U(5)$. Therefore, the solutions of equation (2.2) for the Yukawa couplings are different, as can be seen from equation (4.1), which reflects in the phenomenology, as we will see in the next section.

In the dimensionful sector, the sum rule gives us the following boundary conditions at the GUT scale [46]:

$$
\begin{aligned}
& m_{H_{u}}^{2}+2 m_{\mathbf{1 0}}^{2}=m_{H_{d}}^{2}+m_{\overline{\mathbf{5}}}^{2}+m_{\mathbf{1 0}}^{2}=M^{2} \quad \text { for } \quad \mathbf{A} ; \\
& m_{H_{u}}^{2}+2 m_{\mathbf{1 0}}^{2}=M^{2}, \quad m_{H_{d}}^{2}-2 m_{\mathbf{1 0}}^{2}=-\frac{M^{2}}{3}, \quad m_{\overline{\mathbf{5}}}^{2}+3 m_{\mathbf{1 0}}^{2}=\frac{4 M^{2}}{3} \quad \text { for } \quad \mathbf{B},
\end{aligned}
$$

where we use as free parameters $m_{\overline{\mathbf{5}}} \equiv m_{\overline{\mathbf{5}}_{3}}$ and $m_{\mathbf{1 0}} \equiv m_{\mathbf{1 0}_{3}}$ for the model $\mathbf{A}$, and $m_{\mathbf{1 0}} \equiv m_{\mathbf{1 0}_{3}}$ for $\mathbf{B}$, in addition to $M$.

\section{$5 \quad$ Predictions of low energy parameters}

Since the gauge symmetry is spontaneously broken below $M_{\mathrm{GUT}}$, the finiteness conditions do not restrict the renormalization properties at low energies, and all it remains are boundary conditions on the gauge and Yukawa couplings (4.1), the $h=-M C$ relation, and the soft scalar-mass sum rule (3.1) at $M_{\mathrm{GUT}}$, as applied in the two models. Thus we examine the evolution of these parameters according to their RGEs up to two-loops for dimensionless parameters and at oneloop for dimensionful ones with the relevant boundary conditions. Below $M_{\mathrm{GUT}}$ their evolution is assumed to be governed by the MSSM. We further assume a unique supersymmetry breaking scale $M_{s}$ (which we define as the geometric mean of the stop masses) and therefore below that scale the effective theory is just the SM.

We now present the comparison of the predictions of the four models with the experimental data, see [65] for more details, starting with the heavy quark masses. In Fig. 1 we show the FUTA and FUTB predictions for $M_{\text {top }}$ and $m_{\text {bot }}\left(M_{Z}\right)$ as a function of the unified gaugino mass $M$, for the two cases $\mu<0$ and $\mu>0$. In the value of the bottom mass $m_{\mathrm{bot}}$, we have included the corrections coming from bottom squark-gluino loops and top squarkchargino loops [50]. We give the predictions for the running bottom quark mass evaluated at $M_{Z}, m_{\text {bot }}\left(M_{Z}\right)=2.825 \pm 0.1$ [51], to avoid the large QCD uncertainties inherent for the pole mass. The value of $m_{\text {bot }}$ depends strongly on the sign of $\mu$ due to the above mentioned radiative corrections. For both models $\mathbf{A}$ and $\mathbf{B}$ the values for $\mu>0$ are above the central experimental value, with $m_{\text {bot }}\left(M_{Z}\right) \sim 4.0-5.0 \mathrm{GeV}$. For $\mu<0$, on the other hand, model $\mathbf{B}$ has overlap with the experimental allowed values, $m_{\text {bot }}\left(M_{Z}\right) \sim 2.5-2.8 \mathrm{GeV}$, whereas for model $\mathbf{A}, m_{\text {bot }}\left(M_{Z}\right) \sim 1.5-2.6 \mathrm{GeV}$, there is only a small region of allowed parameter space at two sigma level, and only for large values of $M$. This clearly selects the negative sign of $\mu$.

The predictions for the top quark mass $M_{\text {top }}$ are $\sim 183$ and $\sim 172 \mathrm{GeV}$ in the models $\mathbf{A}$ and $\mathbf{B}$ respectively, as shown in the lower plot of Fig. 1. Comparing these predictions with the most recent experimental value $M_{\mathrm{top}}^{\mathrm{exp}}=(170.9 \pm 1.8) \mathrm{GeV}$ [52], and recalling that the theoretical values for $M_{\text {top }}$ may suffer from a correction of $\sim 4 \%$ [53], we see that clearly model $\mathbf{B}$ is singled out. In addition the value of $\tan \beta$ is found to be $\tan \beta \sim 54$ and $\sim 48$ for models $\mathbf{A}$ and $\mathbf{B}$, respectively. Thus from the comparison of the predictions of the two models with experimental data only FUTB with $\mu<0$ survives.

We now analyze the impact of further low-energy observables on the model FUTB with $\mu<0$. In the case where all the soft scalar masses are universal at the unfication scale, there is 

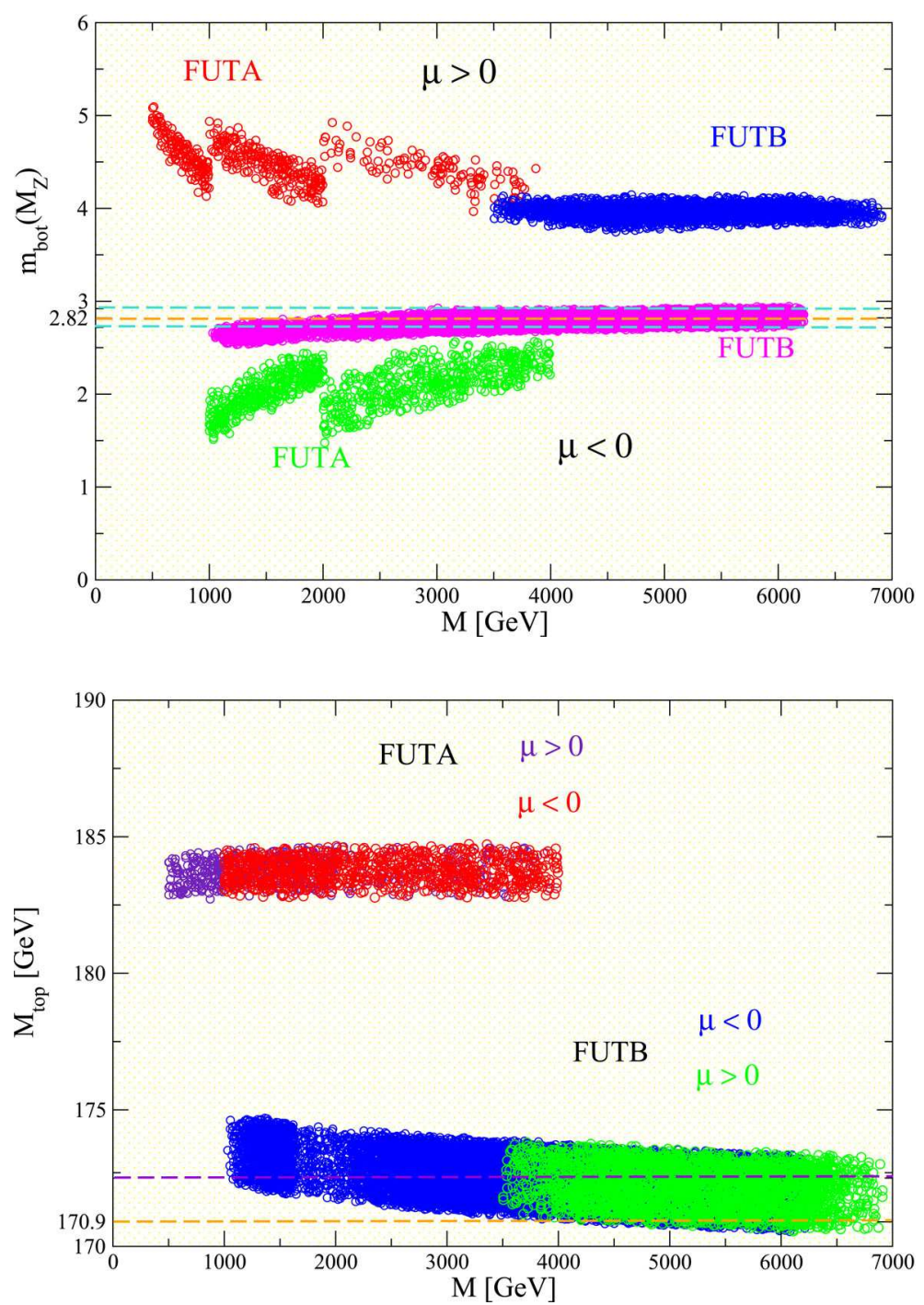

Figure 1. The bottom quark mass at the $Z$ boson scale (upper) and top quark pole mass (lower plot) are shown as function of $M$ for both models.

no region of $M$ below $\mathcal{O}($ few $\mathrm{TeV})$ in which $m_{\tilde{\tau}}>m_{\chi^{0}}$ is satisfied (where $m_{\tilde{\tau}}$ is the lightest $\tilde{\tau}$ mass, and $m_{\chi^{0}}$ the lightest neutralino mass). But once the universality condition is relaxed this problem can be solved naturally, thanks to the sum rule (3.1). Using this equation and imposing the conditions of (a) successful radiative electroweak symmetry breaking, (b) $m_{\tilde{\tau}}^{2}>0$ and (c) $m_{\tilde{\tau}}>m_{\chi^{0}}$, a comfortable parameter space is found for FUTB with $\mu<0$ (and also for FUTA and both signs of $\mu$ ).

As additional constraints we consider the following observables: the rare $b$ decays $\mathrm{BR}(b \rightarrow s \gamma)$ and $\operatorname{BR}\left(B_{s} \rightarrow \mu^{+} \mu^{-}\right)$, the lightest Higgs boson mass as well as the density of cold dark matter in the Universe, assuming it consists mainly of neutralinos. More details and a complete set of references can be found in [65].

For the branching ratio $\mathrm{BR}(b \rightarrow s \gamma)$, we take the present experimental value estimated by the Heavy Flavour Averaging Group (HFAG) is [55]

$$
\operatorname{BR}(b \rightarrow s \gamma)=\left(3.55 \pm 0.24_{-0.10}^{+0.09} \pm 0.03\right) \times 10^{-4}
$$

where the first error is the combined statistical and uncorrelated systematic uncertainty, the latter two errors are correlated systematic theoretical uncertainties and corrections respectively. 


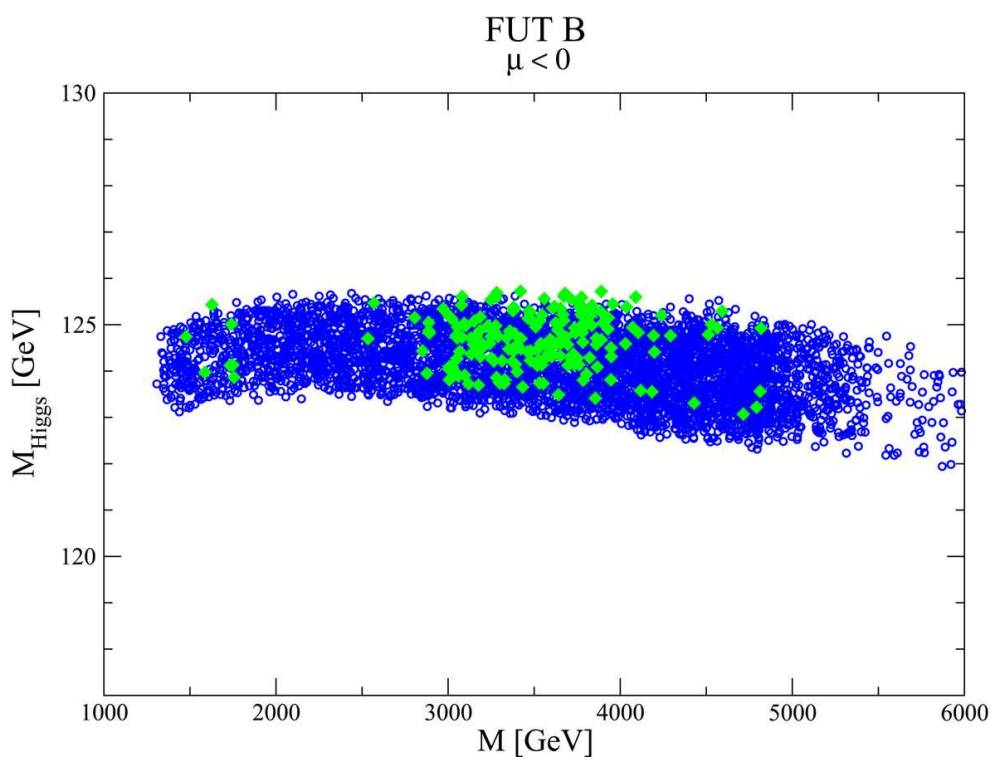

Figure 2. The lightest Higgs mass, $M_{h}$, as function of $M$ for the model FUTB with $\mu<0$, see text.

For the branching ratio $\mathrm{BR}\left(B_{s} \rightarrow \mu^{+} \mu^{-}\right)$, the $\mathrm{SM}$ prediction is at the level of $10^{-9}$, while the present experimental upper limit from the Tevatron is $5.8 \times 10^{-8}$ at the $95 \%$ C.L. [56], providing the possibility for the MSSM to dominate the SM contribution.

Concerning the lightest Higgs boson mass, $M_{h}$, the SM bound of $114.4 \mathrm{GeV}$ [57] can be used. For the prediction we use the code FeynHiggs [58, 59, 60, 61].

The lightest supersymmetric particle (LSP) is an excellent candidate for cold dark matter (CDM) [62], with a density that falls naturally within the range

$$
0.094<\Omega_{\mathrm{CDM}} h^{2}<0.129
$$

favoured by a joint analysis of WMAP and other astrophysical and cosmological data [63].

The prediction for $M_{h}$ of FUTB with $\mu<0$ is shown in Fig. 2. The constraints from the two $B$ physics observables are taken into account. In addition the CDM constraint (evaluated with Micromegas [64]) is fulfilled for the lighter (green) points in the plot, see [65] for details. The lightest Higgs mass ranges in

$$
M_{h} \sim 121-126 \mathrm{GeV},
$$

where the uncertainty comes from variations of the soft scalar masses, and from finite (i.e. not logarithmically divergent) corrections in changing renormalization scheme. To this value one has to add $\pm 3 \mathrm{GeV}$ coming from unknown higher order corrections [60]. We have also included a small variation, due to threshold corrections at the GUT scale, of up to $5 \%$ of the FUT boundary conditions. Thus, taking into account the $B$ physics constraints (and possibly the CDM constraints) results naturally in a light Higgs boson that fulfills the LEP bounds [57].

In the same way the whole SUSY particle spectrum can be derived. The resulting SUSY masses for FUTB with $\mu<0$ are rather large. The lightest SUSY particle starts around $500 \mathrm{GeV}$, with the rest of the spectrum being very heavy. The observation of SUSY particles at the LHC or the ILC will only be possible in very favorable parts of the parameter space. For most parameter combination only a SM-like light Higgs boson in the range of equation (5.1) can be observed.

We note that with such a heavy SUSY spectrum the anomalous magnetic moment of the muon, $(g-2)_{\mu}$ (with $\left.a_{\mu} \equiv(g-2)_{\mu} / 2\right)$, gives only a negligible correction to the SM prediction. 
The comparison of the experimental result and the SM value [66]

$$
a_{\mu}^{\exp }-a_{\mu}^{\text {theo }}=(27.5 \pm 8.4) \times 10^{-10}
$$

would disfavor FUTB with $\mu<0$ by about $3 \sigma$. However, since the SM is not regarded as excluded by $(g-2)_{\mu}$, we still see FUTB with $\mu<0$ as the only surviving model. A more detailed numerical analysis, also using Suspect [67] for the RGE running, and including all theory uncertainties for the models will be presented in a future publication.

\section{Concluding remarks on the Realistic Finite Unified Theories}

The finiteness conditions in the supersymmetric part of the unbroken theory lead to relations among the dimensionless couplings, i.e. gauge-Yukawa unification. In addition the finiteness conditions in the SUSY-breaking sector of the theories lead to a tremendous reduction of the number of the independent soft SUSY-breaking parameters leaving one model (A) with three and another $(\mathbf{B})$ with two free parameters. Therefore the finiteness-constrained MSSM consists of the well known MSSM with boundary conditions at the Grand Unification scale for its various dimensionless and dimensionful parameters inherited from the all-loop finiteness unbroken theories. Obviously these lead to an extremely restricted and, consequently, very predictive parameter space of the MSSM.

\section{Unified Theories from Fuzzy Higher Dimensions}

Coset Space Dimensional Reduction (CSDR) [14, 15, 16, 17, 18, 19, 20, 21, 22] is a unification scheme for obtaining realistic particle models from gauge theories on higher D-dimensional spaces $M^{D}$. It suggests that a unification of the gauge and Higgs sectors of the Standard Model can be achieved in higher than four dimensions. Moreover the addition of fermions in the higher-dimensional gauge theory leads naturally, after CSDR, to Yukawa couplings in four dimensions. We present a study of the CSDR in the non-commutative context which sets the rules for constructing new particle models that might be phenomenologically interesting. One could study CSDR with the whole parent space $M^{D}$ being non-commutative or with just noncommutative Minkowski space or non-commutative internal space. We specialize here to this last situation and therefore eventually we obtain Lorentz covariant theories on commutative Minkowski space. We further specialize to fuzzy non-commutativity, i.e. to matrix type noncommutativity. Thus, following [23], we consider non-commutative spaces like those studied in [5, $6,2]$ and implementing the CSDR principle on these spaces we obtain the rules for constructing new particle models.

Next we reverse the above approach [68] and examine how a four dimensional gauge theory dynamically develops higher dimensions. The very concept of dimension therefore gets an extra, richer dynamical perspective. We present a simple field-theoretical model which realizes the above ideas. It is defined as a renormalizable $S U(N)$ gauge theory on four dimensional Minkowski space $M^{4}$, containing 3 scalars in the adjoint of $S U(N)$ that transform as vectors under an additional global $S O(3)$ symmetry with the most general renormalizable potential. We then show that the model dynamically develops fuzzy extra dimensions, more precisely a fuzzy sphere $S_{N}^{2}$. The appropriate interpretation is therefore as gauge theory on $M^{4} \times S_{N}^{2}$. The lowenergy effective action is that of a four dimensional gauge theory on $M^{4}$, whose gauge group and field content is dynamically determined by compactification and dimensional reduction on the internal sphere $S_{N}^{2}$. An interesting and rich pattern of spontaneous symmetry breaking appears, breaking the original $S U(N)$ gauge symmetry down to either $S U(n)$ or $S U\left(n_{1}\right) \times S U\left(n_{2}\right) \times U(1)$. 
The latter case is the generic one, and implies also a monopole flux induced on the fuzzy sphere. The values of $n_{1}$ and $n_{2}$ are determined dynamically.

We find moreover explicitly the tower of massive Kaluza-Klein modes corresponding to the effective geometry, which justifies the interpretation as a compactified higher-dimensional gauge theory. Nevertheless, the model is renormalizable.

A similar but different mechanism of dynamically generating extra dimensions has been proposed some years ago in [69], known under the name of "deconstruction". In this context, renormalizable four dimensional asymptotically free gauge theories were considered, which develop a "lattice-like" fifth dimension. This idea attracted considerable interest. Our model is quite different, and very simple: The $S U(N)$ gauge theory is shown to develop fuzzy extra dimensions through a standard symmetry breaking mechanism.

\section{The Fuzzy Sphere}

\subsection{Ordinary and Fuzzy spherical harmonics}

Let us start by recalling how to describe fields on the 2 -sphere. The 2 -sphere is a two-dimensional manifold embedded in $\mathbb{R}^{3}$, with a global $S O(3) \sim S U(2)$ isometry group, defined by the equation

$$
x_{1}^{2}+x_{2}^{2}+x_{3}^{2}=R^{2}
$$

for a coordinate basis $x_{\hat{a}}$ in $\mathbb{R}^{3}$. We define the coordinates $x_{\hat{a}}$ in terms of the spherical coordinates $y_{a}=(\theta, \phi)$ and radius $R$ by,

$$
x_{1}=R \sin \theta \cos \phi, \quad x_{2}=R \sin \theta \sin \phi, \quad x_{3}=R \cos \theta,
$$

which dictates the metric of the 2-sphere,

$$
d s^{2}=R^{2} d \theta^{2}+R^{2} \sin ^{2} \theta d \phi^{2} .
$$

The generators of $S U(2) \sim S O(3)$ are the angular momentum operators $L_{i}$,

$$
L_{\hat{a}}=-i \varepsilon_{\hat{a} \hat{b} \hat{c}} x_{\hat{b}} \partial_{\hat{c}} .
$$

In terms of spherical coordinates the angular momentum operators are

$$
L_{1}=i \sin \phi \frac{\partial}{\partial \theta}+i \cos \phi \cot \theta \frac{\partial}{\partial \phi}, \quad L_{2}=-i \cos \phi \frac{\partial}{\partial \theta}+i \sin \phi \cot \theta \frac{\partial}{\partial \phi}, \quad L_{3}=-i \frac{\partial}{\partial \phi},
$$

which we can summarize as

$$
L_{\hat{a}}=-i k_{\hat{a}}^{a} \partial_{a} .
$$

The metric tensor can also be expressed in terms of the Killing vectors $k_{\hat{a}}^{a}$ (defined by the above equations) as

$$
g^{a b}=\frac{1}{R^{2}} k_{\hat{a}}^{a} k_{\hat{a}}^{b} .
$$

We can expand any function on the 2-sphere in terms of the eigenfunctions of the 2-sphere,

$$
a(\theta, \phi)=\sum_{l=0}^{\infty} \sum_{m=-l}^{l} a_{l m} Y_{l m}(\theta, \phi),
$$


where $a_{l m}$ is a complex coefficient and $Y_{l m}(\theta, \phi)$ are the spherical harmonics, which satisfy the equation

$$
L^{2} Y_{l m}=-R^{2} \Delta_{S^{2}} Y_{l m}=l(l+1) Y_{l m}
$$

where $\Delta_{S^{2}}$ is the scalar Laplacian on the 2 -sphere

$$
\Delta_{S^{2}}=\frac{1}{\sqrt{g}} \partial_{a}\left(g^{a b} \sqrt{g} \partial_{b}\right)
$$

The spherical harmonics have an eigenvalue $\mu \sim l(l+1)$ for integer $l=0,1, \ldots$, with degeneracy $2 l+1$. The orthogonality condition of the spherical harmonics is

$$
\int d \Omega Y_{l m}^{\dagger} Y_{l^{\prime} m^{\prime}}=\delta_{l l^{\prime}} \delta_{m m^{\prime}}
$$

where $d \Omega=\sin \theta d \theta d \phi$.

The spherical harmonics can be expressed in terms of the cartesian coordinates $x_{\hat{a}}$ (with $\hat{a}=1,2,3)$ of a unit vector in $\mathbb{R}^{3}$,

$$
Y_{l m}(\theta, \phi)=\sum_{\vec{a}} f_{\hat{a_{1} \ldots \hat{a}_{l}}}^{(l m)} x^{\hat{a_{1}}} \cdots x^{\hat{a_{l}}}
$$

where $f_{\hat{a_{1} \ldots \hat{a}_{l}}}^{(l m)}$ is a traceless symmetric tensor of $S O(3)$ with rank $l$.

Similarly we can expand $N \times N$ matrices on a sphere as,

$$
\hat{a}=\sum_{l=0}^{N-1} \sum_{m=-l}^{l} a_{l m} \hat{Y}_{l m}, \quad \hat{Y}_{l m}=R^{-l} \sum_{\vec{a}} f_{\hat{a_{1}} \ldots \hat{a}_{l}}^{(l m)} \hat{x}^{\hat{a}_{1}} \cdots \hat{x}^{\hat{a}_{l}},
$$

where $\hat{x}_{\hat{a}}=\frac{2 R}{\sqrt{N^{2}-1}} X_{\hat{a}}^{(N)}$ are the generators of $S U(2)$ in the $N$-dimensional representation and $f_{\hat{a}_{1} \ldots \hat{a}_{l}}^{(l m)}$ is the same tensor as in (8.2). The matrices $\hat{Y}_{l m}$ are known as fuzzy spherical harmonics for reasons explained in the next subsection. They obey the orthonormality condition

$$
\operatorname{Tr}_{N}\left(\hat{Y}_{l m}^{\dagger} \hat{Y}_{l^{\prime} m^{\prime}}\right)=\delta_{l l^{\prime}} \delta_{m m^{\prime}}
$$

There is an obvious relation between equations (8.1) and (8.3), namely

$$
\hat{a}=\sum_{l=0}^{N-1} \sum_{m=-l}^{l} a_{l m} \hat{Y}_{l m} \rightarrow a(\theta, \phi)=\sum_{l=0}^{N-1} \sum_{m=-l}^{l} a_{l m} Y_{l m}(\theta, \phi) .
$$

Notice that the expansion in spherical harmonics is truncated at $N-1$ reflecting the finite number of degrees of freedom in the matrix $\hat{a}$. This allows the consistent definition of a matrix approximation of the sphere known as fuzzy sphere.

\subsection{The Matrix Geometry of the fuzzy sphere}

According to the above discussion the fuzzy sphere $[70,2]$ is a matrix approximation of the usual sphere $S^{2}$. The algebra of functions on $S^{2}$ (for example spanned by the spherical harmonics) as explained in the previous section is truncated at a given frequency and thus becomes finite dimensional. The truncation has to be consistent with the associativity of the algebra and this can be nicely achieved relaxing the commutativity property of the algebra. The fuzzy sphere is the "space" described by this non-commutative algebra. The algebra itself is that of $N \times N$ 
matrices. More precisely, the algebra of functions on the ordinary sphere can be generated by the coordinates of $\mathbb{R}^{3}$ modulo the relation $\sum_{\hat{a}=1}^{3} x_{\hat{a}} x_{\hat{a}}=r^{2}$. The fuzzy sphere $S_{N}^{2}$ at fuzziness level $N-1$ is the non-commutative manifold whose coordinate functions $i X_{\hat{a}}$ are $N \times N$ hermitian matrices proportional to the generators of the $N$-dimensional representation of $S U(2)$. They satisfy the condition $\sum_{\hat{a}=1}^{3} X_{\hat{a}} X_{\hat{a}}=\alpha r^{2}$ and the commutation relations

$$
\left[X_{\hat{a}}, X_{\hat{b}}\right]=C_{\hat{a} \hat{b} \hat{c}} X_{\hat{c}}
$$

where $C_{\hat{a} \hat{b} \hat{c}}=\varepsilon_{\hat{a} \hat{b} \hat{c}} / r$ while the proportionality factor $\alpha$ goes as $N^{2}$ for $N$ large. Indeed it can be proven that for $N \rightarrow \infty$ one obtains the usual commutative sphere.

On the fuzzy sphere there is a natural $S U(2)$ covariant differential calculus. This calculus is three-dimensional and the derivations $e_{\hat{a}}$ along $X_{\hat{a}}$ of a function $f$ are given by $e_{\hat{a}}(f)=\left[X_{\hat{a}}, f\right]$. Accordingly the action of the Lie derivatives on functions is given by

$$
\mathcal{L}_{\hat{a}} f=\left[X_{\hat{a}}, f\right]
$$

these Lie derivatives satisfy the Leibniz rule and the $S U(2)$ Lie algebra relation

$$
\left[\mathcal{L}_{\hat{a}}, \mathcal{L}_{\hat{b}}\right]=C_{\hat{a} \hat{b} \hat{c}} \mathcal{L}_{\hat{c}}
$$

In the $N \rightarrow \infty$ limit the derivations $e_{\hat{a}}$ become $e_{\hat{a}}=C_{\hat{a} \hat{b} \hat{c}} x^{\hat{b}} \partial^{\hat{c}}$ and only in this commutative limit the tangent space becomes two-dimensional. The exterior derivative is given by

$$
d f=\left[X_{\hat{a}}, f\right] \theta^{\hat{a}}
$$

with $\theta^{\hat{a}}$ the one-forms dual to the vector fields $e_{\hat{a}},\left\langle e_{\hat{a}}, \theta^{\hat{b}}\right\rangle=\delta_{\hat{a}}^{\hat{b}}$. The space of one-forms is generated by the $\theta^{\hat{a}}$ 's in the sense that for any one-form $\omega=\sum_{i} f_{i} d h_{i} t_{i}$ we can always write $\omega=\sum_{\hat{a}=1}^{3} \omega_{\hat{a}} \theta^{\hat{a}}$ with given functions $\omega_{\hat{a}}$ depending on the functions $f_{i}, h_{i}$ and $t_{i}$. The action of the Lie derivatives $\mathcal{L}_{\hat{a}}$ on the one-forms $\theta^{\hat{b}}$ explicitly reads

$$
\mathcal{L}_{\hat{a}}\left(\theta^{\hat{b}}\right)=C_{\hat{a} \hat{b} \hat{c}} \theta^{\hat{c}}
$$

On a general one-form $\omega=\omega_{\hat{a}} \theta^{\hat{a}}$ we have $\mathcal{L}_{\hat{b}} \omega=\mathcal{L}_{\hat{b}}\left(\omega_{\hat{a}} \theta^{\hat{a}}\right)=\left[X_{\hat{b}}, \omega_{\hat{a}}\right] \theta^{\hat{a}}-\omega_{\hat{a}} C_{\hat{b} \hat{c}}^{\hat{a}} \theta^{\hat{c}}$ and therefore

$$
\left(\mathcal{L}_{\hat{b}} \omega\right)_{\hat{a}}=\left[X_{\hat{b}}, \omega_{\hat{a}}\right]-\omega_{\hat{c}} C_{\hat{b} \hat{a}}^{\hat{c}} ;
$$

this formula will be fundamental for formulating the CSDR principle on fuzzy cosets.

The differential geometry on the product space Minkowski times fuzzy sphere, $M^{4} \times S_{N}^{2}$, is easily obtained from that on $M^{4}$ and on $S_{N}^{2}$. For example a one-form $A$ defined on $M^{4} \times S_{N}^{2}$ is written as

$$
A=A_{\mu} d x^{\mu}+A_{\hat{a}} \theta^{\hat{a}}
$$

with $A_{\mu}=A_{\mu}\left(x^{\mu}, X_{\hat{a}}\right)$ and $A_{\hat{a}}=A_{\hat{a}}\left(x^{\mu}, X_{\hat{a}}\right)$.

One can also introduce spinors on the fuzzy sphere and study the Lie derivative on these spinors. Although here we have sketched the differential geometry on the fuzzy sphere, one can study other (higher-dimensional) fuzzy spaces (e.g. fuzzy $C P^{M}$ ) and with similar techniques their differential geometry. 


\section{Dimensional Reduction of Fuzzy Extra Dimensions}

\subsection{Actions in higher dimensions seen as four-dimensional actions (expansion in Kaluza-Klein modes)}

First we consider on $M^{4} \times(S / R)_{F}$ a non-commutative gauge theory with gauge group $G=U(P)$ and examine its four-dimensional interpretation. $(S / R)_{F}$ is a fuzzy coset, for example the fuzzy sphere $S_{N}^{2}$. The action is

$$
\mathcal{A}_{Y M}=\frac{1}{4 g^{2}} \int d^{4} x k \operatorname{Tr} \operatorname{tr}_{G} F_{M N} F^{M N},
$$

where $k T r$ denotes integration over the fuzzy coset $(S / R)_{F}$ described by $N \times N$ matrices; here the parameter $k$ is related to the size of the fuzzy coset space. For example for the fuzzy sphere we have $r^{2}=\sqrt{N^{2}-1} \pi k$ [2]. In the $N \rightarrow \infty$ limit $k \operatorname{Tr}$ becomes the usual integral on the coset space. For finite $N$, Tr is a good integral because it has the cyclic property $\operatorname{Tr}\left(f_{1} \cdots f_{p-1} f_{p}\right)=\operatorname{Tr}\left(f_{p} f_{1} \cdots f_{p-1}\right)$. It is also invariant under the action of the group $S$, that is infinitesimally given by the Lie derivative. In the action (9.1) $\operatorname{tr}_{G}$ is the gauge group $G$ trace. The higher-dimensional field strength $F_{M N}$, decomposed in four-dimensional space-time and extradimensional components, reads as follows $\left(F_{\mu \nu}, F_{\mu \hat{b}}, F_{\hat{a} \hat{b}}\right)$; explicitly the various components of the field strength are given by

$$
\begin{aligned}
& F_{\mu \nu}=\partial_{\mu} A_{\nu}-\partial_{\nu} A_{\mu}+\left[A_{\mu}, A_{\nu}\right], \\
& F_{\mu \hat{a}}=\partial_{\mu} A_{\hat{a}}-\left[X_{\hat{a}}, A_{\mu}\right]+\left[A_{\mu}, A_{\hat{a}}\right], \\
& F_{\hat{a} \hat{b}}=\left[X_{\hat{a}}, A_{\hat{b}}\right]-\left[X_{\hat{b}}, A_{\hat{a}}\right]+\left[A_{\hat{a}}, A_{\hat{b}}\right]-C_{\hat{a} \hat{b}}^{\hat{c}} A_{\hat{c}} .
\end{aligned}
$$

Under an infinitesimal $G$ gauge transformation $\lambda=\lambda\left(x^{\mu}, X^{\hat{a}}\right)$ we have

$$
\delta A_{\hat{a}}=-\left[X_{\hat{a}}, \lambda\right]+\left[\lambda, A_{\hat{a}}\right],
$$

thus $F_{M N}$ is covariant under local $G$ gauge transformations: $F_{M N} \rightarrow F_{M N}+\left[\lambda, F_{M N}\right]$. This is an infinitesimal Abelian $U(1)$ gauge transformation if $\lambda$ is just an antihermitian function of the coordinates $x^{\mu}, X^{\hat{a}}$ while it is an infinitesimal non-Abelian $U(P)$ gauge transformation if $\lambda$ is valued in $\operatorname{Lie}(U(P))$, the Lie algebra of hermitian $P \times P$ matrices. In the following we will always assume $\operatorname{Lie}(U(P))$ elements to commute with the coordinates $X^{\hat{a}}$. In fuzzy/non-commutative gauge theory and in Fuzzy-CSDR a fundamental role is played by the covariant coordinate,

$$
\varphi_{\hat{a}} \equiv X_{\hat{a}}+A_{\hat{a}} .
$$

This field transforms indeed covariantly under a gauge transformation, $\delta\left(\varphi_{\hat{a}}\right)=\left[\lambda, \varphi_{\hat{a}}\right]$. In terms of $\varphi$ the field strength in the non-commutative directions reads,

$$
F_{\mu \hat{a}}=\partial_{\mu} \varphi_{\hat{a}}+\left[A_{\mu}, \varphi_{\hat{a}}\right]=D_{\mu} \varphi_{\hat{a}}, \quad F_{\hat{a} \hat{b}}=\left[\varphi_{\hat{a}}, \varphi_{\hat{b}}\right]-C_{\hat{a} \hat{b}}^{\hat{c}} \varphi_{\hat{c}}
$$

and using these expressions the action reads

$$
\mathcal{A}_{Y M}=\int d^{4} x \operatorname{Tr} \operatorname{tr}_{G}\left(\frac{k}{4 g^{2}} F_{\mu \nu}^{2}+\frac{k}{2 g^{2}}\left(D_{\mu} \varphi_{\hat{a}}\right)^{2}-V(\varphi)\right),
$$

where the potential term $V(\varphi)$ is the $F_{\hat{a} \hat{b}}$ kinetic term (in our conventions $F_{\hat{a} \hat{b}}$ is antihermitian so that $V(\varphi)$ is hermitian and non-negative)

$$
\begin{aligned}
V(\varphi)= & -\frac{k}{4 g^{2}} \operatorname{Tr}_{G} \operatorname{tr}_{\hat{a} \hat{b}} F_{\hat{a} \hat{b}} F_{\hat{a} \hat{b}} \\
& =-\frac{k}{4 g^{2}} \operatorname{Tr}_{G} \operatorname{tr}_{G}\left(\left[\varphi_{\hat{a}}, \varphi_{\hat{b}}\right]\left[\varphi^{\hat{a}}, \varphi^{\hat{b}}\right]-4 C_{\hat{a} \hat{b} \hat{c}} \varphi^{\hat{a}} \varphi^{\hat{b}} \varphi^{\hat{c}}+2 r^{-2} \varphi^{2}\right) .
\end{aligned}
$$


The action (9.3) is naturally interpreted as an action in four dimensions. The infinitesimal $G$ gauge transformation with gauge parameter $\lambda\left(x^{\mu}, X^{\hat{a}}\right)$ can indeed be interpreted just as an $M^{4}$ gauge transformation. We write

$$
\lambda\left(x^{\mu}, X^{\hat{a}}\right)=\lambda^{\alpha}\left(x^{\mu}, X^{\hat{a}}\right) \mathcal{T}^{\alpha}=\lambda^{h, \alpha}\left(x^{\mu}\right) T^{h} \mathcal{T}^{\alpha},
$$

where $\mathcal{T}^{\alpha}$ are hermitian generators of $U(P), \lambda^{\alpha}\left(x^{\mu}, X^{\hat{a}}\right)$ are $n \times n$ antihermitian matrices and thus are expressible as $\lambda\left(x^{\mu}\right)^{\alpha, h} T^{h}$, where $T^{h}$ are antihermitian generators of $U(n)$. The fields $\lambda\left(x^{\mu}\right)^{\alpha, h}$, with $h=1, \ldots, n^{2}$, are the Kaluza-Klein modes of $\lambda\left(x^{\mu}, X^{\hat{a}}\right)^{\alpha}$. We now consider on equal footing the indices $h$ and $\alpha$ and interpret the fields on the r.h.s. of (9.5) as one field valued in the tensor product Lie algebra $\operatorname{Lie}(U(n)) \otimes \operatorname{Lie}(U(P))$. This Lie algebra is indeed $\operatorname{Lie}(U(n P))$ (the $(n P)^{2}$ generators $T^{h} \mathcal{T}^{\alpha}$ being $n P \times n P$ antihermitian matrices that are linear independent). Similarly we rewrite the gauge field $A_{\nu}$ as

$$
A_{\nu}\left(x^{\mu}, X^{\hat{a}}\right)=A_{\nu}^{\alpha}\left(x^{\mu}, X^{\hat{a}}\right) \mathcal{T}^{\alpha}=A_{\nu}^{h, \alpha}\left(x^{\mu}\right) T^{h} \mathcal{T}^{\alpha},
$$

and interpret it as a $\operatorname{Lie}(U(n P))$ valued gauge field on $M^{4}$, and similarly for $\varphi_{\hat{a}}$. Finally $\operatorname{Tr} \operatorname{tr}_{G}$ is the trace over $U(n P)$ matrices in the fundamental representation.

Up to now we have just performed a ordinary fuzzy dimensional reduction. Indeed in the commutative case the expression (9.3) corresponds to rewriting the initial lagrangian on $M^{4} \times S^{2}$ using spherical harmonics on $S^{2}$. Here the space of functions is finite dimensional and therefore the infinite tower of modes reduces to the finite sum given by $\mathrm{Tr}$.

\subsection{Non-trivial Dimensional reduction in the case of Fuzzy Extra Dimensions}

Next we reduce the number of gauge fields and scalars in the action (9.3) by applying the Coset Space Dimensional Reduction (CSDR) scheme. Since $S U(2)$ acts on the fuzzy sphere $(S U(2) / U(1))_{F}$, and more in general the group $S$ acts on the fuzzy coset $(S / R)_{F}$, we can state the CSDR principle in the same way as in the continuum case, i.e. the fields in the theory must be invariant under the infinitesimal $S U(2)$, respectively $S$, action up to an infinitesimal gauge transformation

$$
\mathcal{L}_{\hat{b}} \phi=\delta_{W_{\hat{b}}} \phi=W_{\hat{b}} \phi, \quad \mathcal{L}_{\hat{b}} A=\delta_{W_{\hat{b}}} A=-D W_{\hat{b}},
$$

where $A$ is the one-form gauge potential $A=A_{\mu} d x^{\mu}+A_{\hat{a}} \theta^{\hat{a}}$, and $W_{\hat{b}}$ depends only on the coset coordinates $X^{\hat{a}}$ and (like $A_{\mu}, A_{a}$ ) is antihermitian. We thus write $W_{\hat{b}}=W_{\hat{b}}^{\alpha} \mathcal{T}^{\alpha}, \alpha=1,2, \ldots, P^{2}$, where $\mathcal{T}^{i}$ are hermitian generators of $U(P)$ and $\left(W_{b}^{i}\right)^{\dagger}=-W_{b}^{i}$, here ${ }^{\dagger}$ is hermitian conjugation on the $X^{\hat{a}}$ 's.

In terms of the covariant coordinate $\varphi_{\hat{d}}=X_{\hat{d}}+A_{\hat{d}}$ and of

$$
\omega_{\hat{a}} \equiv X_{\hat{a}}-W_{\hat{a}}
$$

the CSDR constraints assume a particularly simple form, namely

$$
\begin{aligned}
& {\left[\omega_{\hat{b}}, A_{\mu}\right]=0,} \\
& C_{\hat{b} \hat{d} \hat{e}} \varphi^{\hat{e}}=\left[\omega_{\hat{b}}, \varphi_{\hat{d}}\right] .
\end{aligned}
$$

In addition we have a consistency condition following from the relation $\left[\mathcal{L}_{\hat{a}}, \mathcal{L}_{\hat{b}}\right]=C_{\hat{a} \hat{b}}{ }^{\hat{c}} \mathcal{L}_{\hat{c}}$ :

$$
\left[\omega_{\hat{a}}, \omega_{\hat{b}}\right]=C_{\hat{a} \hat{b}}^{\hat{c}} \omega_{c}
$$

where $\omega_{\hat{a}}$ transforms as $\omega_{\hat{a}} \rightarrow \omega_{\hat{a}}^{\prime}=g \omega_{\hat{a}} g^{-1}$. One proceeds in a similar way for the spinor fields [23]. 


\subsubsection{Solving the CSDR constraints for the fuzzy sphere}

We consider $(S / R)_{F}=S_{N}^{2}$, i.e. the fuzzy sphere, and to be definite at fuzziness level $N-1(N \times N$ matrices). We study here the basic example where the gauge group is $G=U(1)$. In this case the $\omega_{\hat{a}}=\omega_{\hat{a}}\left(X^{\hat{b}}\right)$ appearing in the consistency condition (9.8) are $N \times N$ antihermitian matrices and therefore can be interpreted as elements of $\operatorname{Lie}(U(N))$. On the other hand the $\omega_{\hat{a}}$ satisfy the commutation relations (9.8) of $\operatorname{Lie}(S U(2))$. Therefore in order to satisfy the consistency condition (9.8) we have to embed $\operatorname{Lie}(S U(2))$ in $\operatorname{Lie}(U(N))$. Let $T^{h}$ with $h=1, \ldots,(N)^{2}$ be the generators of $\operatorname{Lie}(U(N))$ in the fundamental representation, we can always use the convention $h=(\hat{a}, u)$ with $\hat{a}=1,2,3$ and $u=4,5, \ldots, N^{2}$ where the $T^{\hat{a}}$ satisfy the $S U(2)$ Lie algebra,

$$
\left[T^{\hat{a}}, T^{\hat{b}}\right]=C^{\hat{a} \hat{b}} T^{\hat{c}}
$$

Then we define an embedding by identifying

$$
\omega_{\hat{a}}=T_{\hat{a}} .
$$

The constraint (9.6), $\left[\omega_{\hat{b}}, A_{\mu}\right]=0$, then implies that the four-dimensional gauge group $K$ is the centralizer of the image of $S U(2)$ in $U(N)$, i.e.

$$
K=C_{U(N)}(S U((2)))=S U(N-2) \times U(1) \times U(1),
$$

where the last $U(1)$ is the $U(1)$ of $U(N) \simeq S U(N) \times U(1)$. The functions $A_{\mu}(x, X)$ are arbitrary functions of $x$ but the $X$ dependence is such that $A_{\mu}(x, X)$ is $\operatorname{Lie}(K)$ valued instead of $\operatorname{Lie}(U(N))$, i.e. eventually we have a four-dimensional gauge potential $A_{\mu}(x)$ with values in Lie $(K)$. Concerning the constraint $(9.7)$, it is satisfied by choosing

$$
\varphi_{\hat{a}}=r \varphi(x) \omega_{\hat{a}}
$$

i.e. the unconstrained degrees of freedom correspond to the scalar field $\varphi(x)$ which is a singlet under the four-dimensional gauge group $K$.

The choice (9.9) defines one of the possible embedding of $\operatorname{Lie}(S U(2))$ in $\operatorname{Lie}(U(N))$ ). For example, we could also embed $\operatorname{Lie}(S U(2))$ in $\operatorname{Lie}(U(N))$ using the irreducible $N$-dimensional rep. of $S U(2)$, i.e. we could identify $\omega_{\hat{a}}=X_{\hat{a}}$. The constraint (9.6) in this case implies that the four-dimensional gauge group is $U(1)$ so that $A_{\mu}(x)$ is $U(1)$ valued. The constraint (9.7) leads again to the scalar singlet $\varphi(x)$.

In general, we start with a $U(1)$ gauge theory on $M^{4} \times S_{N}^{2}$. We solve the CSDR constraint (9.8) by embedding $S U(2)$ in $U(N)$. There exist $p_{N}$ embeddings, where $p_{N}$ is the number of ways one can partition the integer $N$ into a set of non-increasing positive integers [70]. Then the constraint (9.6) gives the surviving four-dimensional gauge group. The constraint (9.7) gives the surviving four-dimensional scalars and equation (9.10) is always a solution but in general not the only one. By setting $\phi_{\hat{a}}=\omega_{\hat{a}}$ we obtain always a minimum of the potential. This minimum is given by the chosen embedding of $S U(2)$ in $U(N)$.

An important point that we would like to stress here is the question of the renormalizability of the gauge theory defined on $M_{4} \times(S / R)_{F}$. First we notice that the theory exhibits certain features so similar to a higher-dimensional gauge theory defined on $M_{4} \times S / R$ that naturally it could be considered as a higher-dimensional theory too. For instance the isometries of the spaces $M_{4} \times S / R$ and $M_{4} \times(S / R)_{F}$ are the same. It does not matter if the compact space is fuzzy or not. For example in the case of the fuzzy sphere, i.e. $M_{4} \times S_{N}^{2}$, the isometries are $S O(3,1) \times S O(3)$ as in the case of the continuous space, $M_{4} \times S^{2}$. Similarly the coupling of a gauge theory defined on $M_{4} \times S / R$ and on $M_{4} \times(S / R)_{F}$ are both dimensionful and have exactly the same dimensionality. On the other hand the first theory is clearly non-renormalizable, while 
the latter is renormalizable (in the sense that divergencies can be removed by a finite number of counterterms). So from this point of view one finds a partial justification of the old hopes for considering quantum field theories on non-commutative structures. If this observation can lead to finite theories too, it remains as an open question.

\section{Dynamical Generation of Extra Dimensions}

Let us now discuss a further development [68] of these ideas, which addresses in detail the questions of quantization and renormalization. This leads to a slightly modified model with an extra term in the potential, which dynamically selects a unique (nontrivial) vacuum out of the many possible CSDR solutions, and moreover generates a magnetic flux on the fuzzy sphere. It also allows to show that the full tower of Kaluza-Klein modes is generated on $S_{N}^{2}$.

\subsection{The four dimensional action}

We start with a $S U(N)$ gauge theory on four dimensional Minkowski space $M^{4}$ with coordinates $y^{\mu}, \mu=0,1,2,3$. The action under consideration is

$$
\mathcal{S}_{Y M}=\int d^{4} y \operatorname{Tr}\left(\frac{1}{4 g^{2}} F_{\mu \nu}^{\dagger} F_{\mu \nu}+\left(D_{\mu} \phi_{a}\right)^{\dagger} D_{\mu} \phi_{a}\right)-V(\phi)
$$

where $A_{\mu}$ are $S U(N)$-valued gauge fields, $D_{\mu}=\partial_{\mu}+\left[A_{\mu}, \cdot\right]$, and

$$
\phi_{a}=-\phi_{a}^{\dagger}, \quad a=1,2,3
$$

are 3 antihermitian scalars in the adjoint of $S U(N)$,

$$
\phi_{a} \rightarrow U^{\dagger} \phi_{a} U
$$

where $U=U(y) \in S U(N)$. Furthermore, the $\phi_{a}$ transform as vectors of an additional global $S O(3)$ symmetry. The potential $V(\phi)$ is taken to be the most general renormalizable action invariant under the above symmetries, which is

$$
\begin{aligned}
V(\phi)= & \operatorname{Tr}\left(g_{1} \phi_{a} \phi_{a} \phi_{b} \phi_{b}+g_{2} \phi_{a} \phi_{b} \phi_{a} \phi_{b}-g_{3} \varepsilon_{a b c} \phi_{a} \phi_{b} \phi_{c}+g_{4} \phi_{a} \phi_{a}\right) \\
& +\frac{g_{5}}{N} \operatorname{Tr}\left(\phi_{a} \phi_{a}\right) \operatorname{Tr}\left(\phi_{b} \phi_{b}\right)+\frac{g_{6}}{N} \operatorname{Tr}\left(\phi_{a} \phi_{b}\right) \operatorname{Tr}\left(\phi_{a} \phi_{b}\right)+g_{7}
\end{aligned}
$$

This may not look very transparent at first sight, however it can be written in a very intuitive way. First, we make the scalars dimensionless by rescaling

$$
\phi_{a}^{\prime}=R \phi_{a}
$$

where $R$ has dimension of length; we will usually suppress $R$ since it can immediately be reinserted, and drop the prime from now on. Now observe that for a suitable choice of $R$,

$$
R=\frac{2 g_{2}}{g_{3}},
$$

the potential can be rewritten as

$$
V(\phi)=\operatorname{Tr}\left(a^{2}\left(\phi_{a} \phi_{a}+\tilde{b} \mathbb{1}\right)^{2}+c+\frac{1}{\tilde{g}^{2}} F_{a b}^{\dagger} F_{a b}\right)+\frac{h}{N} g_{a b} g_{a b}
$$


for suitable constants $a, b, c, \tilde{g}, h$, where

$$
\begin{aligned}
& F_{a b}=\left[\phi_{a}, \phi_{b}\right]-\varepsilon_{a b c} \phi_{c}=\varepsilon_{a b c} F_{c}, \\
& \tilde{b}=b+\frac{d}{N} \operatorname{Tr}\left(\phi_{a} \phi_{a}\right), \quad g_{a b}=\operatorname{Tr}\left(\phi_{a} \phi_{b}\right) .
\end{aligned}
$$

We will omit $c$ from now. Notice that two couplings were reabsorbed in the definitions of $R$ and $\tilde{b}$. The potential is clearly positive definite provided

$$
a^{2}=g_{1}+g_{2}>0, \quad \frac{2}{\tilde{g}^{2}}=-g_{2}>0, \quad h \geq 0,
$$

which we assume from now on. Here $\tilde{b}=\tilde{b}(y)$ is a scalar, $g_{a b}=g_{a b}(y)$ is a symmetric tensor under the global $S O(3)$, and $F_{a b}=F_{a b}(y)$ is a $s u(N)$-valued antisymmetric tensor field which will be interpreted as field strength in some dynamically generated extra dimensions below. In this form, $V(\phi)$ looks like the action of Yang-Mills gauge theory on a fuzzy sphere in the matrix formulation $[71,72,73,74]$. It differs from the potential in $(9.2)$ only by the presence of the first term $a^{2}\left(\phi_{a} \phi_{a}+\tilde{b}\right)^{2}$, which is strongly suggested by renormalization. In fact it is necessary for the interpretation as pure YM action, and we will see that it is very welcome on physical grounds since it dynamically determines and stabilizes a vacuum, which can be interpreted as extra-dimensional fuzzy sphere. In particular, it removes unwanted flat directions.

\subsection{Emergence of extra dimensions and the fuzzy sphere}

The vacuum of the above model is given by the minimum of the potential (10.1). Finding the minimum of the potential is a rather nontrivial task, and the answer depends crucially on the parameters in the potential [68]. The conditions for the global minimum imply that $\phi_{a}$ is a representation of $S U(2)$, with Casimir $\tilde{b}$ (where it was assumed for simplicity $h=0$ ). Then, it is easy to write down a large class of solutions to the minimum of the potential, by noting that any decomposition of $N=n_{1} N_{1}+\cdots+n_{h} N_{h}$ into irreps of $S U(2)$ with multiplicities $n_{i}$ leads to a block-diagonal solution

$$
\phi_{a}=\operatorname{diag}\left(\alpha_{1} X_{a}^{\left(N_{1}\right)}, \ldots, \alpha_{k} X_{a}^{\left(N_{k}\right)}\right)
$$

of the vacuum equations, where $\alpha_{i}$ are suitable constants which will be determined below.

It turns out [68] that there are essentially only 2 types of vacua:

1. Type I vacuum. It is plausible that the solution (10.2) with minimal potential contains only representations whose Casimirs are close to $\tilde{b}$. In particular, let $M$ be the dimension of the irrep whose Casimir $C_{2}(M) \approx \tilde{b}$ is closest to $\tilde{b}$. If furthermore the dimensions match as $N=M n$, we expect that the vacuum is given by $n$ copies of the irrep $(M)$, which can be written as $\phi_{a}=\alpha X_{a}^{(N)} \otimes 1_{n}$ with low-energy gauge group $S U(n)$.

2. Type II vacuum. Consider again a solution (10.2) with $n_{i}$ blocks of size $N_{i}=\tilde{N}+m_{i}$, where $\tilde{N}$ is defined by $\tilde{b}=\frac{1}{4}\left(\tilde{N}^{2}-1\right)$, and assume that $\tilde{N}$ is large and $\frac{m_{i}}{\tilde{N}} \ll 1$. The action is then given by

$$
V(\phi)=\operatorname{Tr}\left(\frac{1}{2 \tilde{g}^{2}} \sum_{i} n_{i} m_{i}^{2} \mathbb{1}_{N_{i}}+O\left(\frac{1}{N_{i}}\right)\right) \approx \frac{1}{2 \tilde{g}^{2}} \frac{N}{k} \sum_{i} n_{i} m_{i}^{2},
$$

where $k=\sum n_{i}$ is the total number of irreps, and the solution can be interpreted in terms of "instantons" (non-Abelian monopoles) on the internal fuzzy sphere [71]. Hence in order to determine the solution of type (10.2) with minimal action, we simply have to minimize 
$\sum_{i} n_{i} m_{i}^{2}$, where the $m_{i} \in \mathbb{Z}-\tilde{N}$ satisfy the constraint $\sum n_{i} m_{i}=N-k \tilde{N}$. In this case the solution with minimal potential among all possible partitions (10.2) is given by

$$
\phi_{a}=\left(\begin{array}{cc}
\alpha_{1} X_{a}^{\left(N_{1}\right)} \otimes 1_{n_{1}} & 0 \\
0 & \alpha_{2} X_{a}^{\left(N_{2}\right)} \otimes 1_{n_{2}}
\end{array}\right),
$$

with low-energy gauge group $S U\left(n_{1}\right) \times S U\left(n_{2}\right) \times U(1)$.

Again, the $X_{a}^{(N)}$ are interpreted as coordinate functions of a fuzzy sphere $S_{N}^{2}$, and the "scalar" action

$$
S_{\phi}=\operatorname{Tr} V(\phi)=\operatorname{Tr}\left(a^{2}\left(\phi_{a} \phi_{a}+\tilde{b}\right)^{2}+\frac{1}{\tilde{g}^{2}} F_{a b}^{\dagger} F_{a b}\right)
$$

for $N \times N$ matrices $\phi_{a}$ is precisely the action for a $U(n)$ Yang-Mills theory on $S_{N}^{2}$ with coupling $\tilde{g}$, as shown in [71]. In fact, the new term $\left(\phi_{a} \phi_{a}+\tilde{b}\right)^{2}$ is essential for this interpretation, since it stabilizes the vacuum $\phi_{a}=X_{a}^{(N)}$ and gives a large mass to the extra "radial" scalar field which otherwise arises. The fluctuations of $\phi_{a}=X_{a}^{(N)}+A_{a}$ then provide the components $A_{a}$ of a higher-dimensional gauge field $A_{M}=\left(A_{\mu}, A_{a}\right)$, and the action can be interpreted as YM theory on the 6-dimensional space $M^{4} \times S_{N}^{2}$, with gauge group depending on the particular vacuum. We therefore interpret the vacuum as describing dynamically generated extra dimensions in the form of a fuzzy sphere $S_{N}^{2}$. This geometrical interpretation can be fully justified by working out the spectrum of Kaluza-Klein modes. The effective low-energy theory is then given by the zero modes on $S_{N}^{2}$. This approach provides a clear dynamical selection of the geometry due to the term $\left(\phi_{a} \phi_{a}+\tilde{b}\right)^{2}$ in the action.

Perhaps the most remarkable aspect of this model is that the geometric interpretation and the corresponding low-energy degrees of freedom depend in a nontrivial way on the parameters of the model, which are running under the RG group. Therefore the massless degrees of freedom and their geometrical interpretation depend on the energy scale. In particular, the low-energy gauge group generically turns out to be $S U\left(n_{1}\right) \times S U\left(n_{2}\right) \times U(1)$ or $S U(n)$, while gauge groups which are products of more than two simple components (apart from $U(1)$ ) do not seem to occur. The values of $n_{1}$ and $n_{2}$ are determined dynamically, and with the appropriate choice of parameters it is possible to construct vacuum solutions where they are as small, such as 2 and $3[68]$.

It is interesting to examine the running of the coupling constants under the RG. $R$ turns out to run only logarithmically, implies that the scale of the internal spheres is only mildly affected by the RG flow. However, $\tilde{b}$ is running essentially quadratically, hence is generically large. This is quite welcome here: starting with some large $N, \tilde{b} \approx C_{2}(\tilde{N})$ must indeed be large in order to lead to the geometric interpretation discussed above. Hence the problems of naturalness or fine-tuning appear to be rather mild here.

A somewhat similar model has been studied in [75, 76], which realizes deconstruction and a "twisted" compactification of an extra fuzzy sphere based on a supersymmetric gauge theory. Our model is different and does not require supersymmetry, leading to a much richer pattern of symmetry breaking and effective geometry. For other relevant work see e.g. [6].

The dynamical formation of fuzzy spaces found here is also related to recent work studying the emergence of stable submanifolds in modified IIB matrix models. In particular, previous studies based on actions for fuzzy gauge theory different from ours generically only gave results corresponding to $U(1)$ or $U(\infty)$ gauge groups, see e.g. [77, 78, 79] and references therein. The dynamical generation of a nontrivial index on noncommutative spaces has also been observed in $[80,81]$ for different models.

Our mechanism may also be very interesting in the context of the recent observation [82] that extra dimensions are very desirable for the application of noncommutative field theory to 
particle physics. Other related recent work discussing the implications of the higher-dimensional point of view on symmetry breaking and Higgs masses can be found in [83, 84, 85, 86]. These issues could now be discussed within a renormalizable framework.

\section{Concluding remarks on the use of Fuzzy extra dimensions}

Non-commutative Geometry has been regarded as a promising framework for obtaining finite quantum field theories and for regularizing quantum field theories. In general quantization of field theories on non-commutative spaces has turned out to be much more difficult and with less attractive ultraviolet features than expected, see however [87] and [88]. Recall also that non-commutativity is not the only suggested tool for constructing finite field theories. Indeed four-dimensional finite gauge theories have been constructed in ordinary space-time and not only those which are $\mathcal{N}=4$ and $\mathcal{N}=2$ supersymmetric, and most probably phenomenologically uninteresting, but also chiral $\mathcal{N}=1$ gauge theories [28] which already have been successful in predicting the top quark mass and have rich phenomenology that could be tested in future colliders $[28,24,29,46,53,54,48]$. In the present work we have not addressed the finiteness of non-commutative quantum field theories, rather we have used non-commutativity to produce, via Fuzzy-CSDR, new particle models from particle models on $M^{4} \times(S / R)_{F}$.

A major difference between fuzzy and ordinary SCDR is that in the fuzzy case one always embeds $S$ in the gauge group $G$ instead of embedding just $R$ in $G$. This is due to the fact that the differential calculus on the fuzzy coset space is based on $\operatorname{dim} S$ derivations instead of the restricted $\operatorname{dim} S-\operatorname{dim} R$ used in the ordinary one. As a result the four-dimensional gauge group $H=C_{G}(R)$ appearing in the ordinary CSDR after the geometrical breaking and before the spontaneous symmetry breaking due to the four-dimensional Higgs fields does not appear in the Fuzzy-CSDR. In Fuzzy-CSDR the spontaneous symmetry breaking mechanism takes already place by solving the Fuzzy-CSDR constraints. The four-dimensional potential has the typical "mexican hat" shape, but it appears already spontaneously broken. Therefore in four dimensions appears only the physical Higgs field that survives after a spontaneous symmetry breaking. Correspondingly in the Yukawa sector of the theory we have the results of the spontaneous symmetry breaking, i.e. massive fermions and Yukawa interactions among fermions and the physical Higgs field. Having massive fermions in the final theory is a generic feature of CSDR when $S$ is embedded in $G$ [15]. We see that if one would like to describe the spontaneous symmetry breaking of the SM in the present framework, then one would be naturally led to large extra dimensions.

A fundamental difference between the ordinary CSDR and its fuzzy version is the fact that a non-Abelian gauge group $G$ is not really required in high dimensions. Indeed the presence of a $U(1)$ in the higher-dimensional theory is enough to obtain non-Abelian gauge theories in four dimensions.

In a further development, we have presented a renormalizable four dimensional $S U(N)$ gauge theory with a suitable multiplet of scalars, which dynamically develops fuzzy extra dimensions that form a fuzzy sphere. The model can then be interpreted as 6-dimensional gauge theory, with gauge group and geometry depending on the parameters in the original Lagrangian. We explicitly find the tower of massive Kaluza-Klein modes, consistent with an interpretation as compactified higher-dimensional gauge theory, and determine the effective compactified gauge theory. This model has a unique vacuum, with associated geometry and low-energy gauge group depending only on the parameters of the potential.

There are many remarkable aspects of this model. First, it provides an extremely simple and geometrical mechanism of dynamically generating extra dimensions, without relying on subtle dynamics such as fermion condensation and particular Moose- or Quiver-type arrays of gauge groups and couplings, such as in [69] and following work. Rather, our model is based on a basic 
lesson from noncommutative gauge theory, namely that noncommutative or fuzzy spaces can be obtained as solutions of matrix models. The mechanism is quite generic, and does not require fine-tuning or supersymmetry. This provides in particular a realization of the basic ideas of compactification and dimensional reduction within the framework of renormalizable quantum field theory. Moreover, we are essentially considering a large $N$ gauge theory, which should allow to apply the analytical techniques developed in this context.

In particular, it turns out that the generic low-energy gauge group is given by $S U\left(n_{1}\right) \times$ $S U\left(n_{2}\right) \times U(1)$ or $S U(n)$, while gauge groups which are products of more than two simple components (apart from $U(1)$ ) do not seem to occur in this model. The values of $n_{1}$ and $n_{2}$ are determined dynamically. Moreover, a magnetic flux is induced in the vacua with non-simple gauge group, which is very interesting in the context of fermions, since internal fluxes naturally lead to chiral massless fermions. This will be studied in detail elsewhere.

There is also an intriguing analogy between our toy model and string theory, in the sense that as long as $a=0$, there are a large number of possible vacua (given by all possible partitions) corresponding to compactifications, with no dynamical selection mechanism to choose one from the other. Remarkably this analog of the "string vacuum problem" is simply solved by adding a term to the action.

\section{Acknowledgments}

G.Z. would like to thank the organizers for the warm hospitality. This work is supported by the EPEAEK programmes "Pythagoras" and co-funded by the European Union (75\%) and the Hellenic state (25\%); also supported in part by the mexican grant PAPIIT-UNAM IN115207.

\section{References}

[1] Connes A., Non-commutative geometry, Academic Press, San Diego, 1994.

[2] Madore J., An introduction to non-commutative differential geometry and its physical applications, London Mathematical Society Lecture Note Series, Vol. 257, Cambridge University Press, Cambridge, 1999.

[3] Connes A., Lott J., Particle models and noncommutative geometry (expanded version), Nuclear Phys. B Proc. Suppl. 18 (1991), 29-47.

[4] Martin C.P., Gracia-Bondia M.J., Varilly J.C., The standard model as a noncommutative geometry: the low-energy regime, Phys. Rep. 294 (1998) 363-406, hep-th/9605001.

[5] Dubois-Violette M., Madore J., Kerner R., Gauge bosons in a noncommutative geometry, Phys. Lett. B 217 (1989), 485-488.

Dubois-Violette M., Madore J., Kerner R., Classical bosons in a noncommutative geometry, Classical Quantum Gravity 6 (1989), 1709-1724.

Dubois-Violette M., Kerner R., Madore J., Noncommutative differential geometry and new models of gauge theory, J. Math. Phys. 31 (1990), 316-323.

[6] Madore J., On a quark-lepton duality, Phys. Lett. B 305 (1993) 84-89.

Madore J., On a noncommutative extension of electrodynamics, in Proceedings "Spinors, Twistors, Clifford Algebras and Quantum Deformations" (1992, Wroclaw), 1992, 285-298, hep-ph/9209226.

[7] Connes A., Douglas M.R., Schwarz A., Non-commutative geometry and matrix theory: compactification on tori, J. High Energy Phys. 1998 (1998), no. 2, 003, 41 pages, hep-th/9711162.

[8] Seiberg N., Witten E., String theory and noncommutative geometry, J. High Energy Phys. 1999 (1999), no. 9, 032, 99 pages, hep-th/9908142.

[9] Chaichian M., Presnajder P., Sheikh-Jabbari M.M., Tureanu A., Noncommutative standard model: model building, Eur. Phys. J. C 29 (2003), 413-432, hep-th/0107055.

[10] Calmet X., Jurco B., Schupp P., Wess J., Wohlgenannt M., The standard model on non-commutative spacetime, Eur. Phys. J. C 23 (2002), 363-376, hep-ph/011111.

Aschieri P., Jurco B., Schupp P., Wess J., Non-commutative GUTs, standard model and C, P, T, Nuclear Phys. B 651 (2003), 45-70, hep-th/0205214. 
[11] Jurco B., Schraml S., Schupp P., Wess J., Enveloping algebra valued gauge transformations for non-Abelian gauge groups on non-commutative spaces, Eur. Phys. J. C 17 (2000), 521-526, hep-th/0006246.

Jurco B., Schupp P., Wess J., Non-Abelian noncommutative gauge theory via noncommutative extra dimensions, Nuclear Phys. B 604 (2001), 148-180, hep-th/0102129.

Jurco B., Moller L., Schraml S., Schupp S., Wess J., Construction of non-Abelian gauge theories on noncommutative spaces, Eur. Phys. J. C 21 (2001), 383-388, hep-th/0104153.

Barnich G., Brandt F., Grigoriev M., Seiberg-Witten maps and noncommutative Yang-Mills theories for arbitrary gauge groups, J. High Energy Phys. 2002 (2002), no. 8, 023, 12 pages, hep-th/0206003.

[12] Brandt F., Martin C.P., Ruiz F.R., Anomaly freedom in Seiberg-Witten noncommutative gauge theories, J. High Energy Phys. 2003 (2003), no. 07, 068, 32 pages, hep-th/0307292.

[13] Carlson C.E., Carone C.D., Lebed R.F., Bounding noncommutative QCD, Phys. Lett. B 518 (2001), 201206, hep-ph/0107291.

Behr W., Deshpande N.G., Duplancic G., Schupp P., Trampetic J., Wess J., The $Z \rightarrow \gamma \gamma, g g$ decays in the noncommutative standard model, Eur. Phys. J. C 29 (2003), 441-446, hep-ph/0202121.

Hinchliffe I., Kersting N., Ma Y.L., Review of the phenomenology of noncommutative geometry, Internat. J. Modern Phys. A 19 (2004), 179-205, hep-ph/0205040.

Schupp P., Trampetic J., Wess J., Raffelt G., The photon neutrino interaction in non-commutative gauge field theory and astrophysical bounds, Eur. Phys. J. C 36 (2004), 405-410, hep-ph/0212292.

[14] Forgacs P., Manton N.S., Space-time symmetries in gauge theories, Comm. Math. Phys. 72 (1980), 15-35.

[15] Kapetanakis D., Zoupanos G., Coset space dimensional reduction of gauge theories, Phys. Rep. 219 (1992), $1-76$.

[16] Kubyshin Y.A., Volobuev I.P., Mourao J.M., Rudolph G., Dimensional reduction of gauge theories, spontaneous compactification and model building, Lecture Notes in Physics, Vol. 349, Springer Verlag, Heidelberg, 1989.

[17] Bais F.A., Barnes K.J., Forgacs P., Zoupanos G., Dimensional reduction of symmetric gauge theories, in, Proceedings "High Energy Physics, HEP1985" (1985, Bari), 1985, 60-62.

Forgacs P., Lust D., Zoupanos G., Physics from multidimensional gauge theories?, in Proceedings of the 2nd Hellenic School on Elementary Particle Physics (September 1-20, 1985, Corfu, Greece), Editors E.N. Argyres and G. Zoupanos, World Scientific, 1986, 26 pages.

Zoupanos G., Farakos K., Kapetanakis D., Koutsoumbas G., Coset space dimensional reduction approach to the standard model, in Proceedings "Electroweak Interactions and Unified Theories (1988, Les Arcs), 1988, 559-565.

Zoupanos G., Farakos K., Kapetanakis D., Koutsoumbas G., Dimemsional reduction of gauge theories, in Proceedings "ICHEP 1988", 1988, 1137-1141.

Zoupanos G., Coset space dimensional reduction of gauge theories, in Proceedings of Warsaw Symposium (1991), 1991, 446-460.

[18] Chapline G., Manton N.S., The geometrical significance of certain Higgs potentials: an approach to grand unification, Nuclear Phys. B 184 (1981), 391-405.

Bais F.A., Barnes K.J., Forgacs P., Zoupanos G., Dimensional reduction of gauge theories yielding unified models spontaneously broken to $S U(3) \times U(1)$, Nuclear Phys. B 263 (1986), 557-590.

Farakos K., Koutsoumbas G., Surridge M., Zoupanos G., Dimensional reduction and the Higgs potential, Nuclear Phys. B 291 (1987), 128-140.

Farakos K., Koutsoumbas G., Surridge M., Zoupanos G., Geometrical hierarchy and spontaneous symmetry breaking, Phys. Lett. B 191 (1987), 135-140.

Kubyshin Yu.A., Mourao J.M., Volobuev I.P., Scalar fields in the dimensional reduction scheme for symmetric spaces, Internat. J. Modern Phys. A 4 (1989), 151-171.

[19] Manton N.S., Fermions and parity violation in dimensional reduction schemes, Nuclear Phys. B 193 (1981), $502-516$.

Chapline G., Slansky R., Dimensional reduction and flavor chirality, Nuclear Phys. B 209 (1982), 461-483.

[20] Wetterich C., Dimensional reduction of Weyl, Majorana and Majorana-Weyl spinors, Nuclear Phys. B 222 (1983), 20-44.

Palla L., On dimensional reduction of gauge theories: symmetric fields and harmonic expansion, Z. Phys. C 24 (1984), 195-204.

Pilch K., Schellekens A.N., Formulae for the eigenvalues of the Laplacian on tensor harmonics on symmetric coset spaces, J. Math. Phys. 25 (1984), 3455-3459.

Forgacs P., Horvath Z., Palla L., Spontaneous compactification to nonsymmetric spaces, Z. Phys. C 30 (1986), 261-266.

Barnes K.J., Forgacs P., Surridge M., Zoupanos G., On fermion masses in a dimensional reduction scheme, Z. Phys. C 33 (1987), 427-431. 
[21] Farakos K., Kapetanakis D., Koutsoumbas G., Zoupanos G., The standard model from a gauge theory in ten-dimensions via CSDR Phys. Lett. B 211 (1988), 322-328. Hanlon B.E., Joshi G.C., A three generation unified model from coset space dimensional reduction, Phys. Lett. B 298 (1993), 312-317.

[22] Manousselis P., Zoupanos G., Dimensional reduction over coset spaces and supersymmetry breaking, J. High Energy Phys. 2002 (2002), no. 3, 002, 32 pages, hep-ph/0111125.

Manousselis P., Zoupanos G., Soft supersymmetry breaking due to dimensional reduction over nonsymmetric coset spaces, Phys. Lett. B 518 (2001), 171-180, hep-ph/0106033.

Manousselis P., Zoupanos G., Supersymmetry breaking by dimensional reduction over coset spaces, Phys. Lett. B 504 (2001), 122-130, hep-ph/0010141.

Manousselis P., Zoupanos G., Dimensional reduction of ten-dimensional supersymmetric gauge theories in the $N=1, D=4$ superfield formalism, J. High Energy Phys. 2004 (2004), no. 11, 025, 24 pages, hep-ph/0406207.

Manousselis P., Prezas N., Zoupanos G., Supersymmetric compactifications of heterotic strings with fluxes and condensates, Nuclear Phys. B 739 (2006), 85-105, hep-th/0511122.

Chatzistavrakidis A., Manousselis P., Prezas N., Zoupanos G., On the consistency of coset space dimensional reduction, arXiv:0708.3222.

[23] Aschieri P., Madore J., Manousselis P., Zoupanos G., Dimensional reduction over fuzzy coset spaces, J. High Energy Phys. 2004 (2004), 034, 21 pages, hep-th/0310072.

Aschieri P., Madore J., Manousselis P., Zoupanos G., Unified theories from fuzzy extra dimensions, Fortsch. Phys. 52 (2004), 718-723, hep-th/0401200.

Aschieri P., Madore J., Manousselis P., Zoupanos G., Renormalizable theories from fuzzy higher dimensions, hep-th/0503039.

[24] Kubo J., Mondragón M., Zoupanos G., Reduction of couplings and heavy top quark in the minimal SUSY GUT, Nuclear Phys. B 424 (1994), 291-307.

[25] Zimmermann W., Reduction in the number of coupling parameters, Comm. Math. Phys. 97 (1985), 211-225. Oehme R., Zimmermann W., Relation between effective couplings for asymptotically free models, Comm. Math. Phys. 97 (1985), 569-582.

Ma E., Modified quantum chromodynamics: exact global color symmetry and asymptotic freedom, Phys. Rev. D 17 (1978), 623-628.

Ma E., Fixing the Higgs boson mass, Phys. Rev. D 31 (1985), 1143-1145.

[26] Lucchesi C., Piguet O., Sibold K., Vanishing $\beta$-functions in $N=1$ supersymmetric gauge theories, Helv. Phys. Acta 61 (1988), 321-344.

Piguet O., Sibold K., Nonrenormalization theorems of chiral anomalies and finiteness in supersymmetric Yang-Mills theories, Internat. J. Modern Phys. A 1 (1986), 913-942.

Piguet O., Sibold K., Non-renormalization theorems of chiral anomalies and finiteness, Phys. Lett. B 177 (1986), 373-376.

Lucchesi C., Zoupanos G., All order finiteness in $N=1 \mathrm{SYM}$ theories: criteria and applications, Fortsch. Phys. 45 (1997), 129-143, hep-ph/9604216.

[27] Ermushev A., Kazakov D., Tarasov O., Finite $N=1$ supersymmetric grand unified theories, Nuclear Phys. B 281 (1987), 72-84.

Kazakov D., Finite $N=1$ SUSY gauge field theories, Modern Phys. Lett. A 2 (1987), 663-674.

[28] Kapetanakis D., Mondragon M., Zoupanos G., Finite unified models, Z. Phys. C 60 (1993), 181-186, hep-ph/9210218.

Mondragon M., Zoupanos G., Finite unified theories and the top quark mass, Nuclear Phys. C Proc. Suppl. 37 (1995), 98-105.

[29] Kubo J., Mondragón M., Zoupanos G., Perturbative unification of soft supersymmetry breaking terms Phys. Lett. B 389 (1996), 523-532, hep-ph/9609218.

[30] Jack I., Jones D.R.T., Renormalization group invariance and universal soft supersymmetry breaking, Phys. Lett. B 349 (1995), 294-299, hep-ph/9501395.

[31] Rajpoot S., Taylor J.G., On finite quantum field theories, Phys. Lett. B 147 (1984), 91-95.

[32] Rajpoot S., Taylor J.G., Towards finite quantum field theories, Internat. J. Theoret. Phys. 25 (1986), 117-138.

[33] Parkes A., West P., Finiteness in rigid supersymmetric theories, Phys. Lett. B 138 (1984), 99-104.

Jones D., Mezincescu L., The chiral anomaly and a class of two loop finite supersymmetric gauge theories, Phys. Lett. B 138 (1984), 293-295.

[34] Jones D.R.T., Mezincescu L., Yao Y.-P., Soft breaking of two loop finite $N=1$ supersymmetric gauge theories, Phys. Lett. B 148 (1984), 317-322. 
[35] Jack I., Jones D.R.T., Soft supersymmetry breaking and finiteness, Phys. Lett. B 333 (1994), 372-379, hep-ph/9405233.

[36] Leigh R., Strassler M., Exactly marginal operators and duality in four-dimensional $N=1$ supersymmetric gauge theory, Nuclear Phys. B 447 (1995), 95-136, hep-th/9503121.

[37] Novikov V., Shifman M., Vainstein A., Zakharov V., Instanton effects in supersymmetric theories, $N u$ clear Phys. B 229 (1983), 407-420.

Novikov V., Shifman M., Vainstein A., Zakharov V., Beta function in supersymmetric gauge theories: instantons versus traditional approach, Phys. Lett. B 166 (1986), 329-333.

Shifman M., Little miracles of supersymmetric evolution of gauge couplings, Internat. J. Modern Phys. A 11 (1996), 5761-5784, hep-ph/9606281.

[38] Avdeev L.V., Kazakov D.I., Kondrashuk I.N., Renormalizations in softly broken SUSY gauge theories, Nuclear Phys. B 510 (1998), 289-312, hep-ph/9709397.

Kazakov D.I., Exploring softly broken SUSY theories via Grassmannian Taylor expansion, Phys. Lett. B 449 (1999), 201-206, hep-ph/9812513.

[39] Kobayashi T., Kubo J., Zoupanos G., Further all loop results in softly broken supersymmetric gauge theories, Phys. Lett. B 427 (1998), 291-299, hep-ph/9802267.

Kobayashi T. et al., Finite and gauge-Yukawa unified theories: theory and predictions, AIP Conf. Proc. 490 (1999), 279-309.

[40] Yamada Y., Two loop renormalization group equations for soft SUSY breaking scalar interactions: supergraph method, Phys. Rev. D 50 (1994), 3537-3545, hep-ph/9401241.

[41] Hisano J., Shifman M., Exact results for soft supersymmetry breaking parameters in supersymmetric gauge theories, Phys. Rev. D 56 (1997), 5475-5482, hep-ph/9705417.

[42] Kawamura T., Kobayashi T., Kubo J., Soft scalar mass sum rule in gauge Yukawa unified models and its superstring interpretation, Phys. Lett. B 405 (1997), 64-70, hep-ph/9703320.

[43] Delbourgo R., Superfield perturbation theory and renormalization, Nuovo Cimento A 25 (1975), 646-656. Salam A., Strathdee J.A., Feynman rules for superfields, Nuclear Phys. B 86 (1975), 142-152.

Fujikawa K., Lang W., Perturbation calculations for the scalar multiplet in a superfield formulation, Nuclear Phys. B 88 (1975), 61-76.

Grisaru M.T., Rocek M., Siegel W., Improved methods for supergraphs, Nuclear Phys. B 159 (1979), 429450 .

[44] Girardello L., Grisaru M., Soft breaking of supersymmetry, Nuclear Phys. B 194 (1982), 65-76.

[45] Jack I., Jones D.R.T., Pickering A., Renormalization invariance and the soft beta functions, Phys. Lett. B 426 (1998), 73-77, hep-ph/9712542.

[46] Kobayashi T., Kubo J., Mondragón M., Zoupanos G., Constraints on finite soft supersymmetry breaking terms, Nuclear Phys. B 511 (1998), 45-68, hep-ph/9707425.

Kobayashi T., Kubo J., Mondragón M., Zoupanos G., Exact finite and gauge-Yukawa unified theories and their predictions, Acta Phys. Polon. B 30 (1999) 2013-2027.

Mondragón M., Zoupanos G., Higgs mass prediction in finite unified theories, Acta Phys. Polon. B 34 (2003), 5459-5468.

[47] Jones D.R.T., Raby S., A two loop finite supersymmetric $S U(5)$ theory: towards a theory of fermion masses, Phys. Lett. B 143 (1984), 137-141.

[48] Babu K.S., Enkhbat T., Gogoladze I., Finite grand unified theories and the quark mixing matrix, Phys. Lett. B 555 (2003), 238-247, hep-ph/0204246.

[49] Ma E., Mondragón M., Zoupanos G., Finite $S U(N)^{k}$ unification, J. High Energy Phys. 2004 (2004), no. 12, 026, 14 pages, hep-ph/0407236.

[50] Carena M., Garcia D., Nierste U., Wagner C., Effective Lagrangian for the $\overline{t b H}^{+}$interaction in the MSSM and charged Higgs phenomenology, Nuclear Phys. B 577 (2000), 88-120, hep-ph/9912516.

[51] Erler J., Private communication.

[52] Tevatron Electroweak Working Group, hep-ex/0703034.

[53] Kubo J., Mondragón M., Zoupanos G., Testing gauge Yukawa unified models by M(t), Nuclear Phys. B 479 (1996), 25-45, hep-ph/9512435.

[54] Kobayashi T., Kubo J., Mondragón M., Zoupanos G., Finite unification, Surveys High Energ. Phys. 16 (2001), 87-129. 
[55] Barate R. et al. [ALEPH Collaboration], A measurement of the inclusive $b \rightarrow s \gamma$ branching ratio, Phys. Lett. B 429 (1998), 169-187.

Chen S. et al. [CLEO Collaboration], Branching fraction and photon energy spectrum for $b \rightarrow s \gamma$, Phys. Rev. Lett. 87 (2001), 251807, 11 pages, hep-ex/0108032.

Koppenburg P. et al. [Belle Collaboration], An inclusive measurement of the photon energy spectrum in $b \rightarrow s \gamma$ decays, Phys. Rev. Lett. 93 (2004), 061803, 6 pages, hep-ex/0403004.

[56] Herndon M., Searches for FCNC Decays $\mathbf{B}_{\mathbf{s}(\mathbf{d})} \rightarrow \mu^{+} \mu-$, Talk given at ICHEP04 (August, 2004, Beijing), available at http://ichep04.ihep.ac.cn/db/paper.php.

[57] LEP Higgs working group, Search for the standard model Higgs boson at LEP, Phys. Lett. B 565 (2003), 61-75, hep-ex/0306033.

Schael S. et al. [ALEPH Collaboration], Search for neutral MSSM Higgs bosons at LEP, Eur. Phys. J. C 47 (2006), 547-587, hep-ex/0602042.

[58] Heinemeyer S., Hollik W., Weiglein G., FeynHiggs: a program for the calculation of the masses of the neutral CP-even Higgs bosons in the MSSM, Comput. Phys. Comm. 124 (2000), 76-89, hep-ph/9812320, see http://www.feynhiggs.de/.

[59] Heinemeyer S., Hollik W., Weiglein G., The masses of the neutral CP - even Higgs bosons in the MSSM: accurate analysis at the two loop level, Eur. Phys. J. C 9 (1999), 343-366, hep-ph/9812472.

[60] Degrassi G., Heinemeyer S., Hollik W., Slavich P., Weiglein G., Towards high precision predictions for the MSSM Higgs sector, Eur. Phys. J. C 28 (2003), 133-143, hep-ph/0212020.

[61] Frank M., Hahn T., Heinemeyer S., Hollik W., Rzehak H., Weiglein G., The Higgs boson masses and mixings of the complex MSSM in the Feynman-diagrammatic approach, J. High Energy Phys. 2007 (2007), no. 2, 047, 56 pages, hep-ph/0611326.

[62] Goldberg H., Constraint on the photino mass from cosmology, Phys. Rev. Lett. 50 (1983), 1419-1422. Ellis J., Hagelin J., Nanopoulos D., Olive K., Srednicki M., Supersymmetric relics from the big bang, Nuclear Phys. B 238 (1984), 453-476.

[63] Bennett C. et al., First year Wilkinson microwave anisotropy probe (WMAP) observations: preliminary maps and basic results, Astrophys. J. Suppl. 148 (2003), 1-42, astro-ph/0302207.

Spergel D. et al. [WMAP Collaboration], First year Wilkinson microwave anisotropy probe (WMAP) observations: determination of cosmological parameters, Astrophys. J. Suppl. 148 (2003), 175-194, astro-ph/0302209.

[64] Belanger G., Boudjema F., Pukhov A., Semenov A., micrOMEGAs: a program for calculating the relic density in the MSSM, Comput. Phys. Comm. 149 (2002), 103-120, hep-ph/0112278.

Belanger G., Boudjema F., Pukhov A., Semenov A., micrOMEGAs: version 1.3, Comput. Phys. Comm. 174 (2006), 577-604, hep-ph/0405253.

[65] Heinemeyer S., Mondragón M., Zoupanos G., Confronting finite unified theories with low-energy phenomenology, arXiv:0712.3630.

[66] Davier M., The hadronic contribution to $(g-2)_{\mu}$, Nuclear Phys. Proc. Suppl. 169 (2007), 288-296, hep-ph/0701163.

[67] Djouadi A., Kneur J., Moultaka G., SuSpect: a Fortran code for the supersymmetric and Higgs particle spectrum in the MSSM, Comput. Phys. Comm. 176 (2007) 426-455, hep-ph/0211331.

[68] Aschieri P., Grammatikopoulos T., Steinacker H., Zoupanos G., Dynamical generation of fuzzy extra dimensions, dimensional reduction and symmetry breaking, J. High Energy Phys. 2006 (2006), no. 9, 026, 26 pages, hep-th/0606021.

Steinacker H., Zoupanos G., Fermions on spontaneously generated spherical extra dimensions, J. High Energy Phys. 2007 (2007), no. 9, 017, 34 pages, arXiv:0706.0398.

[69] Arkani-Hamed N., Cohen A.G., Georgi H., (De)constructing dimensions, Phys. Rev. Lett. 86 (2001), 47574761, hep-th/0104005.

[70] Madore J., The fuzzy sphere, Classical Quantum Gravity 9 (1992), 69-88.

[71] Steinacker H., Quantized gauge theory on the fuzzy sphere as random matrix model, Nuclear Phys. B 679 (2004), 66-98, hep-th/0307075.

[72] Steinacker H., Gauge theory on the fuzzy sphere and random matrices, Springer Proc. Phys. 98 (2005), 307-311, hep-th/0409235.

[73] Carow-Watamura U., Watamura S., Noncommutative geometry and gauge theory on fuzzy sphere, Comm. Math. Phys. 212 (2000), 395-413, hep-th/9801195. 
[74] Presnajder P., Gauge fields on the fuzzy sphere, Modern Phys. Lett. A 18 (2003), 2431-2438.

[75] Andrews R.P., Dorey N., Spherical deconstruction, Phys. Lett. B 631 (2005), 74-82, hep-th/0505107.

[76] Andrews R.P., Dorey N., Deconstruction of the Maldacena-Nunez compactification, Nuclear Phys. B 751 (2006), 304-341, hep-th/0601098.

[77] Azuma T., Nagao K., Nishimura J., Perturbative dynamics of fuzzy spheres at large N, J. High Energy Phys. 2005 (2005), no. 6, 081, 16 pagegs, hep-th/0410263.

[78] Azuma T., Bal S., Nishimura J., Dynamical generation of gauge groups in the massive Yang-Mills-ChernSimons matrix model, Phys. Rev. D 72 (2005), 066005, 4 pages, hep-th/0504217.

[79] Azuma T., Bal S., Nagao K., Nishimura J., Nonperturbative studies of fuzzy spheres in a matrix model with the Chern-Simons term, J. High Energy Phys. 2004 (2004), no. 5, 005, 35 pages, hep-th/0401038.

[80] Aoki H., Iso S., Maeda T., Nagao K., Dynamical generation of a nontrivial index on the fuzzy 2-sphere, Phys. Rev. D 71 (2005), 045017, 30 pages, Erratum, Phys. Rev. D 71 (2005), 069905, hep-th/0412052.

[81] Aoki H., Nishimura J., Susaki Y., Suppression of topologically nontrivial sectors in gauge theory on $2 d$ non-commutative geometry, J. High Energy Phys. 2007 (2007), no. 10, 024, 14 pages, hep-th/0604093.

[82] Abel S.A., Jaeckel J., Khoze V.V., Ringwald A., Noncommutativity, extra dimensions, and power law running in the infrared, J. High Energy Phys. 2006 (2006), no. 1, 105, 23 pages, hep-ph/0511197.

[83] Lim C.S., Maru N., Hasegawa K., Six dimensional gauge-Higgs unification with an extra space $S^{2}$ and the hierarchy problem, hep-th/0605180.

[84] Dvali G.R., Randjbar-Daemi S., Tabbash R., The origin of spontaneous symmetry breaking in theories with large extra dimensions, Phys. Rev. D 65 (2002), 064021, 27 pages, hep-ph/0102307.

[85] Antoniadis I., Benakli K., Quiros M., Supersymmetry and electroweak breaking by extra dimensions, Acta Phys. Polon. B 33 (2002), 2477-2488.

[86] Scrucca C.A., Serone M., Silvestrini L., Electroweak symmetry breaking and fermion masses from extra dimensions, Nuclear Phys. B 669 (2003), 128-158, hep-ph/0304220.

[87] Grosse H., Wulkenhaar R., Renormalisation of $\phi^{4}$-theory on noncommutative $R^{4}$ to all orders, hep-th/0403232.

[88] Steinacker H., Quantized gauge theory on the fuzzy sphere as random matrix model, Nuclear Phys. B 679 (2004), 66-98, hep-th/0307075.

Grosse H., Steinacker H., Finite gauge theory on fuzzy $C P^{2}$, Nuclear Phys. $B 707$ (2005), 145-198, hep-th/0407089. 